\title{
Disambiguation of social polarization concepts and measures
}

\section{Aaron Bramson, Patrick Grim, Daniel J. Singer, Steven Fisher, William Berger, Graham Sack \& Carissa Flocken}

To cite this article: Aaron Bramson, Patrick Grim, Daniel J. Singer, Steven Fisher, William Berger, Graham Sack \& Carissa Flocken (2016) Disambiguation of social polarization concepts and measures, The Journal of Mathematical Sociology, 40:2, 80-111, DOI: 10.1080/0022250X.2016.1147443

To link to this article: https://doi.org/10.1080/0022250X.2016.1147443

\section{Published online: 13 Apr 2016.}

\section{Submit your article to this journal ๘}

山ll Article views: 635

Q View related articles ¿

View Crossmark data $₫$

4

Citing articles: 12 View citing articles $\asymp$ 


\title{
Disambiguation of social polarization concepts and measures
}

\author{
Aaron Bramson ${ }^{\mathrm{a}, \mathrm{b}}$, Patrick Grimc, Daniel J. Singer ${ }^{\mathrm{d}}$, Steven Fisher ${ }^{\mathrm{e}, \mathrm{f}}$, William Berger ${ }^{\mathrm{e}, \mathrm{f}}$, \\ Graham Sack ${ }^{\mathrm{g}}$, and Carissa Flocken ${ }^{\mathrm{h}}$ \\ aRiken Brain Science Institute, Laboratory for Symbolic Cognitive Development, Wakoshi, Japan; ${ }^{b}$ Department of \\ General Economics, Ghent University, Ghent, Belgium; 'Department of Philosophy, Group for Logic \& Formal \\ Semantics, Stony Brook University, Stony Brook, New York, USA; dPhilosophy Department, University of \\ Pennsylvania, Philadelphia, Pennsylvania, USA; ${ }^{e}$ Center for the Study of Complex Systems, University of Michigan, \\ Ann Arbor, Michigan, USA; fDepartment of Political Science, University of Michigan, Ann Arbor, Michigan, USA; \\ 9Department of English \& Comparative Literature, Columbia University, New York, New York, USA; ${ }^{\text {hH} H o n o r s ~ P r o g r a m, ~}$ \\ University of Michigan, Ann Arbor, Michigan, USA
}

\begin{abstract}
This article distinguishes nine senses of polarization and provides formal measures for each one to refine the methodology used to describe polarization in distributions of attitudes. Each distinct concept is explained through a definition, formal measures, examples, and references. We then apply these measures to GSS data regarding political views, opinions on abortion, and religiosity-topics described as revealing social polarization. Previous breakdowns of polarization include domain-specific assumptions and focus on a subset of the distribution's features. This has conflated multiple, independent features of attitude distributions. The current work aims to extract the distinct senses of polarization and demonstrate that by becoming clearer on these distinctions we can better focus our efforts on substantive issues in social phenomena.
\end{abstract}

\section{ARTICLE HISTORY}

Received 25 January 2015

Revised 16 January 2016

Accepted 22 January 2016

\section{KEYWORDS}

Belief aggregation; formal epistemology; measurement; polarization; social epistemology

\section{Introduction}

Though the term "polarization" is frequently invoked to describe patterns in distributions (e.g., in opinion polls), it is not the single concept it is often taken to be. Occurrences of polarization frequently appear in the sociology and political science literature (Baldassarri \& Gelman, 2008; Bartels, 2000; Brownstein, 2007; DiMaggio, Evans, \& Bryson, 1996; Fiorina, Abrams, \& Pope, 2010; Hetherington \& Weiler, 2009; Hunter, 1991; McCarty, Poole, \& Rosenthal, 2008), but the specific ways in which polarization is realized are not systematically distinguished. Often entire articles appear on the topic of polarization, but with little attempt to make it clear what precisely is meant by the term. In those cases in which a formal measure is provided (see Christen, Ott, \& Schwarz, 2013; DiMaggio et al., 1996; Fiorina et al., 2010; Estaban \& Ray 2012), it may be specific to the analyzed dataset or topic of interest, not fully formalized, not evaluated compared to other measures and/or presented as a measure that is a composite of the measures presented here. Because a genuine understanding of the phenomena at issue demands making definitions unambiguous, here we tease out nine distinct concepts of polarization and demonstrate how each one can be made precise with an example formal measure.

In this effort we make no claims of completeness; to the contrary, we acknowledge that alternative measures are possible within each concept and point toward further concepts beyond the nine included here. Specifically, we are elsewhere pursuing measures that incorporate spatial and social network configurations, but our focus here is on polarization phenomena within a distribution of

CONTACT Aaron Bramson bramson@brain.riken.jp E Laboratory for Symbolic Cognitive Development, RIKEN Brain Science Institute, BSI West Building 2F, 2-1 Hirosawa, Wako City, Saitama 351-0198 Japan

Color versions of one or more of the figures in the article can be found online at www.tandfonline.com/gmas. 
cardinal property values. One common example of appropriate data in the social sciences is the Likert scale responses of one's degree of agreement in a survey statement. These properties are variably referred to as "attitudes," "beliefs," "opinions," "views," "tastes," "ideas," "attributes," and "properties," etc., in the literature, but we refer to all these as attitudes throughout except when matching the terminology from the literature. Furthermore, nonattitudinal properties such as wealth, educational attainment, and commuting distance are also viable targets of the polarization measures presented here. All the measures provided in this article are presented for one-dimensional applications, though they often have obvious equivalents for high-dimensional data. ${ }^{1}$

Examples of data appropriate for the analysis presented here include opinions toward abortion (see Figure 1), people's political views, opinions toward women's rights, degree of support for a particular policy, online product reviews, and the level of funding appropriate for a budget's line item. The abortion figure is illustrative of the ambiguity in polarization phenomena. There are two clear clumps of attitudes at 1 and 5 which indicates some sort of polarization. The groups are largely steady in size and position, so whatever polarization there is, it appears to be constant. If the groups moved further apart, then intuitively the polarization has increased-that is an easy case. What about a scenario in which the liberal group shrinks and the moderate group increases in size? Or how would the level of polarization change if both groups shrank and a sizable third group formed at 8 ? A full characterization of polarization needs to have clear and justifiable answers to these questions.

Our first purpose is to emphasize that polarization refers to a concept cluster rather than single concept. In some senses polarization depends only on the distribution of attitudes across the whole population, while in other senses polarization depends upon how groups within the population are distributed. When groups are appropriate/necessary, we provide clear formal measures in terms of both distributions of attitudes across an unstructured population with endogenously identified

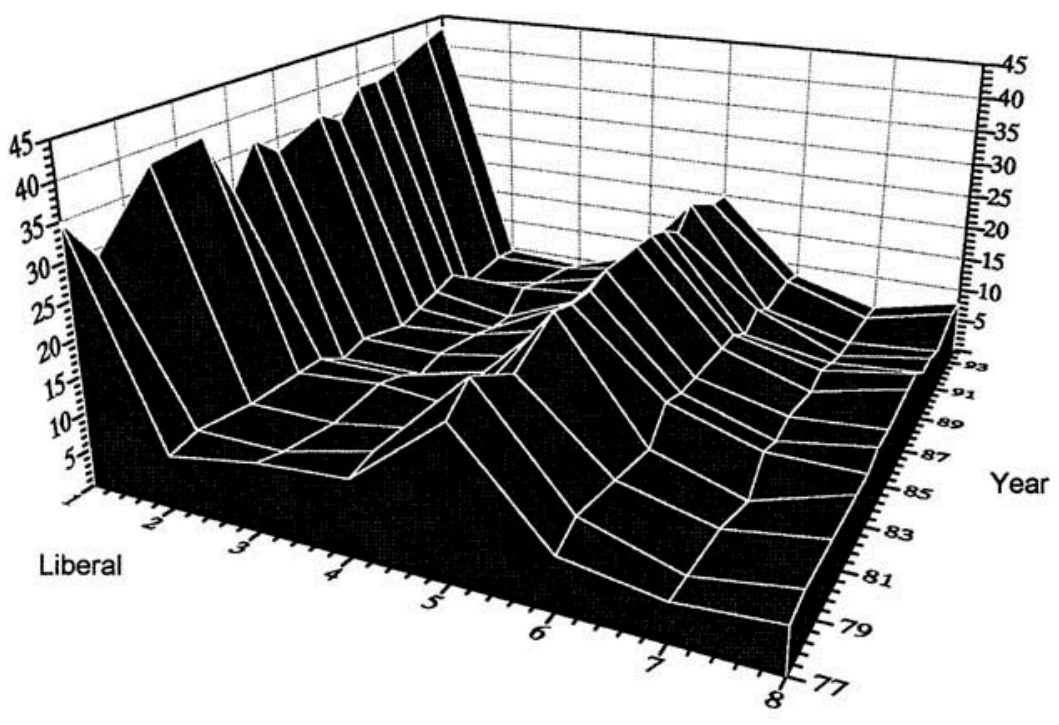

Conservative

Figure 1. Attitudes toward abortion, distribution by year, from the full sample General Social Survey 1977-1994 (DiMaggio et al., 1996).

\footnotetext{
${ }^{1}$ Furthermore, increasing the number of dimensions also expands polarization into additional distinct senses; i.e., there are types of polarization (such as the correlation of attitudes across multiple issue dimensions (Baldassarri \& Gelman, 2008; Fiorina et al., 2010)) that are only sensible for higher-dimensional data.
} 
groupings and distributions of attitudes across exogenously defined groups of individuals. Before moving on to applications we also briefly consider compound concepts that require combining multiple senses; a consideration that is especially important when addressing a literature that commonly combines or switches the meaning of these terms.

In the final section we analyze data regarding people's political views and views toward abortion from the General Social Survey (GSS). These datasets are analyzed by themselves and through the lens of religiosity. Religiosity is categorized by using individuals' response to a question regarding the origin of the Bible. We investigate each sense of polarization for these data by applying our example formal measures and providing a detailed report of the results. We also compare the polarization level of political views and abortion opinions to demonstrate the use of our distinctions for both analyzing trends and differences across issues.

\section{What is polarization?}

This project has three arms: conceptual analysis, formal methodology, and applied social science. The analytical aspect comes into play as we try to better understand the concept(s) of polarization. The scenario in Figure 1 has been identified as a clear case of polarization by DiMaggio et al. (1996) and others. We must ask: "if this distribution is polarized, what makes it polarized?" What does it mean for the distribution to be polarized? What feature(s) of the distribution is the property of polarization picking out? Even if we can all agree and consistently pick out the cases of polarized distributions based on our intuitions, if we cannot explain what makes it polarized (or what it means to be polarized) then we do not have a strong understanding of the phenomena. Furthermore, even for cases as clear as the abortion data, we do not all agree-and most cases are not nearly so clear.

The term polarization is sometimes used to refer to a static property and sometimes a process (DiMaggio et al., 1996). The measures we provide here are properties of a distribution that can be captured at any time-slice of the system. The numbers produced by our measures are not intended to be informative in isolation; rather, they gain their meaning as relative values through comparisons (i.e., being more or less polarized). They can therefore be used to compare two different questions at a given time (e.g., are attitudes about education more or less polarized than attitudes about abortion) or they can be made into measures of a polarizing process through the standard approach of measuring the change in value across time (polarization in trends).

The comparative approach allows us to apply our concepts and measures across issues or time without requiring new concepts or measures (Dandekar, Goel, \& Lee, 2013). Because the individual measures are themselves not comparative, calculations can be done independently and then later compared across data, researchers, topics, or domains. A further corollary of our quantitative measurement approach is that when authors say of a system that it has "become polarized" this simply means that the value for some measure(s) has gone from a low to a comparatively high value. That is, it is a quantitative increase rather than being a qualitative shift implying that it possesses a new property.

\section{Formal measures for polarization}

This article focuses on properties distributed on a normalized spectrum along one dimension. These properties can be anything that admits cardinal values because our formalism only depends on features of the distribution and not on what that distribution represents. As stated above, we have chosen to refer to the properties with the catch-all term attitudes, but they are also commonly referred to as beliefs, opinions, views, attributes, and various other terms in the literature. We adopt the simplest method for representing the distribution of attitudes: creating a histogram of the number of individuals holding a specific attitude value along the spectrum. This representation naturally captures the Likert scale data recovered from surveys and opinion polls. It can also be used to capture more open-ended questions (e.g., "what percentage of the federal budget should be spent on education?") and household income values through binning or density functions. 
The definitions for each of the nine senses below are provided along with example measures. The definitions are conceptual distinctions that stand on their own, while the measures are mathematical means to identify those conceptual distinctions in histograms of attitude distributions along a spectrum. $^{2}$ Although some of the particular measures provided here will not work in capturing the distinct senses of polarization in every type of data (e.g., categorical or overly coarse-grained data), they should provide clarity on the conceptual distinctions and are applicable to two key forms of data: histograms of attitude distributions from surveys and simulation outputs of models with epistemic agents.

For concreteness, the measures provided here assume the following characteristics. There are $N$ agents, participants, or respondents: $a_{1}, a_{2}, \ldots a N$. Each agent $a_{i}$ has an attitude value $x_{i}$ on a range normalized to between 0 and 1 ; this is the location of that agent's attitude along the spectrum. The full distribution of all agents' attitudes is written $X$. The set of bins used for discrete characterizations is $R$. For $r \in R$, the expression $y\left(x_{r}\right)$ is the count of agents occupying the bin containing the value $x_{r}$; that is, the number of agents holding attitude $x_{r}$.

\subsection{Spread}

The simplest concept of polarization is the width of the field of attitudes actually represented in the system. Without taking account of the shape of the distribution, or even whether there is continuity between the extremes, the further out the furthest individuals are the more varied their attitudes. The wider the difference in the most extreme views held, the more polarized the population's ideas are (in this sense).

In a modeled range of attitudes, polarization in the sense of spread could be measured as the attitude level of the agent with the highest attitude value minus the attitude level of the agent with the lowest attitude value. This measure is also commonly called the range of the data

$$
\text { spread }=\max _{x_{i}} X-\min _{x_{i}} X
$$

For multidimensional data, the diameter of the system (the greatest pairwise distance among all the points) is the general measure of spread, of which this is a special case.

Polarization in the sense of spread is illustrated in Figure 2 with two groups (red and blue), although the measure is clearly not group-dependent. To the contrary, the measure just described is purposefully inclusive of the whole population; however, seeing a comparison between the situations (a) and (b) in which the groups are largely similar but differ in their spread should aid in grasping this as an isolated sense of polarization. Also, although the diagram represents the height of the distributions, that is also irrelevant for the measure of spread.

An example application would consider the responses to questions such as "What percentage of the federal budget should be spent on education?" and "What percentage of the federal budget
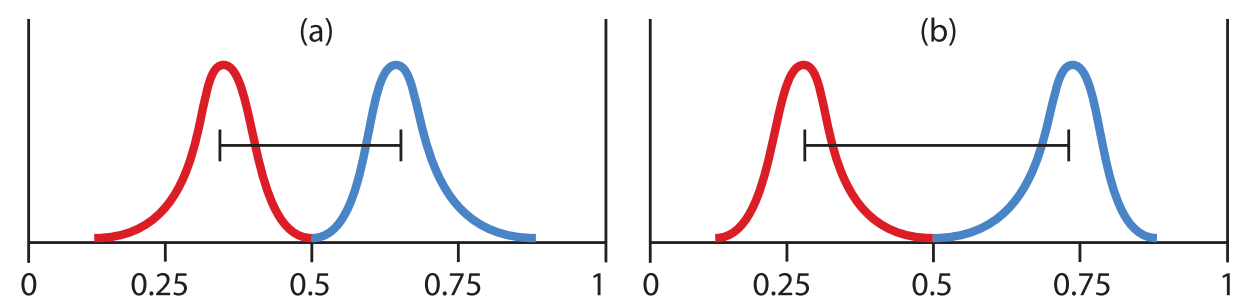

Figure 2. Distribution (b) shows greater polarization in the sense of spread than does distribution (a).

\footnotetext{
${ }^{2}$ Specifically, we provide measures that operate on a metric space so that there is a clear ordering from one end to the other and that distance measures are well-defined everywhere.
} 
should be spent on infrastructure?" For example, spread could be used to compare the results across years, between political affiliations, or among nations. Comparing the widths of the band of held attitudes provides a relative measure, even if a simple one, of polarization on that question. For cases in which data are sparse or incomplete, this may be the only reliable quantitative measure available. The spread measure will often be correlated with the later measures such as the dispersion, coverage, and the distance between group means because many distributions shapes constrain how these values can change. However, for each of the other measures here an example can be generated that demonstrates its independence (see Section 4 for more information about measure independence).

\subsection{Dispersion}

Another simple, and common, measure of the variation in attitudes is statistical dispersion (or statistical variation). Unlike spread, which considers only the extremes of the population, dispersion considers the shape of the whole distribution. Dispersion can increase when groups move apart, or when the distribution flattens, or when the agents within the distribution move away from the middle toward opposite ends of the distribution. Just as in spread, greater dispersion (ceteris paribus) implies higher levels of polarization.

There are many measures of statistical dispersion that are appropriate for our demonstration application, including mean difference, average absolute deviation, standard deviation, coefficient of variation, and entropy are all candidates. For simplicity we select average absolute deviation from the mean as our example here because this can be calculated from aggregate survey data. In our agent-based modeling research, we also find that the mean pairwise value differences are useful. For a population with $\mathrm{N}$ individuals the absolute deviation measure (normalized to be between zero and one) becomes

$$
\text { dispersion }=\frac{2}{N} \sum_{x_{i}}^{N}\left|x_{i}-\bar{X}\right|,
$$

in which $\bar{X}$ is the mean value of distribution $X$. Changes in dispersion for different shape changes are presented in Figure 3. Note that the diagrams in the figure show dispersion increasing as spread is held constant. Like spread, dispersion does not include any notion of groups or subpopulations because it operates on the entire distribution $X$. We address dispersion within groups in Section 3.8 on group consensus.
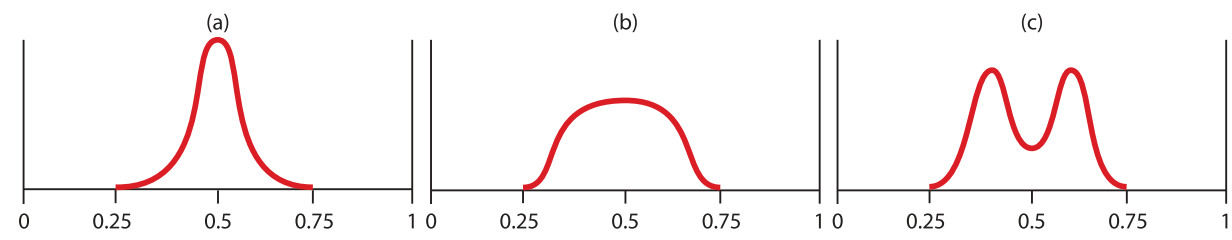

Figure 3. Distribution (c) shows greater polarization in the sense of dispersion than does distribution (b), which is greater than distribution (a).

Various measures of dispersion have already been invoked in both the polarization and statistical literature. DiMaggio et al. (1996) refer to dispersion as "the event that opinions are diverse, 'far apart' in content." Just being "far apart" is not as precise as it could be because although spread is also a measure of statistical dispersion, spread and dispersion as defined here are clearly distinct polarization concepts.

\subsection{Coverage}

Polarization in the sense of coverage captures the level of variation in the values held. One can think of it as the number of distinct attitudes held or the variety of attitudes that at least one person in the 
population holds. Although this sense is not sensitive to the shape of the distribution or even the number of agents who hold each position, it does capture a basic feature of variation. For this sense it does not matter how different the values are, or whether they are clumped together or at extremes; polarization as coverage captures only the variety of distinct attributes in the population. Because more coverage implies more different ideas, and this variety is at odds with converging ideas, polarization is higher.

It is important to keep in mind that this interpretation of polarization as increasing with increasing coverage is in isolation of any other features of the distribution. It is possible to imagine a scenario with two sharp spikes separated by a wide gap. Covering some of the gap with new ideas would make convergence easier via some particular mechanisms. However, this measure is independent of where the coverage occurs, and more coverage could also be created by adding more extreme ideas. So, it is only in isolation that greater coverage tends to imply greater polarization. A more nuanced understanding of a particular case's overall polarization will require multiple measures and further specifics.

A simple way to measure coverage in a discrete (binned data) spectrum is to simply calculate the proportion of bins that are occupied by at least one agent. Note that this discrete measure does not rely on any distance measure or ordering of the values, and therefore is also appropriate for categorical data.

$$
\text { coverage }_{d}=\frac{1}{|R|} \sum_{r \in R} \begin{cases}1 & y\left(x_{r}\right)>0 \\ 0 & y\left(x_{r}\right)=0\end{cases}
$$

That discrete measure, though simple, depends on the choice of bins when applied to nondiscrete data: varying the width and/or location of bins can alter the measure for the same underlying continuous (or fine-grained) data. We also present a continuous measure that does not rely on binning the data. This is done by setting halos of radius $\rho$ around each agent-for one-dimensional data this makes an agent-centered "bin" of width $2 \rho$. Any portion of the attitude space within $\rho$ of an agent is considered covered, with the rest considered uncovered. Continuous coverage is measured by calculating the proportion of the space covered by those halos as

$$
2 \rho+\sum_{x_{i}<x_{j}}^{X} \begin{cases}x_{j}-x_{i} & x_{j}<x_{i}+\rho \\ r+x_{j}-x_{i} & x_{i}+r \leq x_{j} \leq x_{i}+2 \rho \\ 2 \rho & x_{j}>x_{i}+2 \rho\end{cases}
$$

In some applications a further condition should be added for attitude values within $\rho$ of the attitude space boundaries.

Figure 4 illustrates a case in which coverage changes while spread remains constant. Although the height of the distribution (the number of individuals possessing that value) as well as three separate groups (red, blue, and green) are shown in the figure, those details are for reference and irrelevant for measuring coverage.

Coverage is actually a crude measure of diversity, and there is a linkage between a diversity of attitudes and those attitudes being polarized (explored in other work). Though crude, coverage is valuable for categorical data, especially when data are sparse (i.e., the number of observations is small
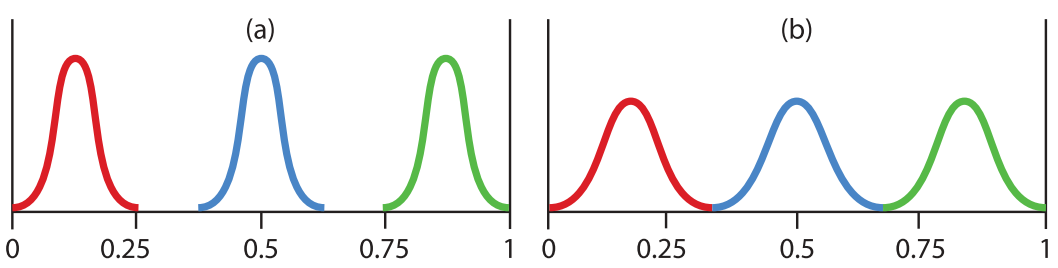

Figure 4. Distribution (b) is more polarized than (a) in the sense of coverage because more of the space is non-empty. 
relative to the number of bins/categories). The continuous version is more valuable when using large datasets for which fine resolution is appropriate and for which the selection of bins biases the results. When applying to higher dimensions, both the continuous and discrete versions can be applied (although some normalization scheme should be implemented for the discrete version to ensure comparability across the number of dimensions).

Coverage does not capture the clustering of the data points, which is a separate polarization concept likely crucial for many broader polarization applications. However, when comparing single- to multidimensional data, coverage is a convenient way to determine the degree to which pairs of attitudes line up. In the simulation literature, the influential paper of Hegselmann and Krause use "consensus (conformity)" to refer to minimal coverage, "polarization(polarity)" to refer to a scenario in which exactly two attitudes are held, and "fragmentation(plurality)" to indicate that multiple attitudes are held within the population (Hegselmann \& Krause, 2002). Coverage converts this categorical breakdown into a measure. Discrete coverage as measured by Eq. (3) is also an accurate measure of polarization for Axelrod's Dissemination of Culture model precisely because it is categorical, even though he invokes some of the other concepts below when defining the term (Axelrod 1997).

\subsection{Regionalization}

In addition to the amount of space covered by attitudes, patterns in the empty attitude space are also relevant for capturing polarization. The number of uncovered ideas is the same concept as coverage (and its measure is the additive inverse). However, the number of uncovered intervals (ignoring their size) is a distinct sense of variety and hence a different way that distributions can be polarized. The more empty regions of the attitude space, the greater the polarization is in the sense of regionalization. Essentially this is reporting a simple version of clustering in the data in which gaps in the attributes indicate separate clusters.

In order to measure the regionalization on a discrete spectrum, it is sufficient to count the regions of contiguous empty bins (including the initial space and trailing space if appropriate). For a discrete spectrum with ordered bins $R$ we can calculate the regionalization with the following formalism:

$$
\sum_{r_{i} \in R} \begin{cases}1 & y\left(r_{0}\right)=0 \\ 1 & y\left(r_{i}\right)>0 \text { and } y\left(r_{i+1}\right)=0 \\ 0 & \text { otherwise }\end{cases}
$$

One can also define a continuous version of this measure using halos, just as we did for coverage, which better accounts for gaps in dense datasets but depends on the choice of radius.

Figure 5 shows a scenario in which regionalization is greater despite the same coverage and spread. The figure has three color-coded subdistributions, and there is a clear connection between empty regions and identifying distinct groups, but that is not the current sense of polarization (both group identification and distinctness are discussed below as separate senses). What this diagram makes clear is that in (a) the subdistributions on the left are clustered together and as a result the distribution is less polarized than in (b) when they are not.
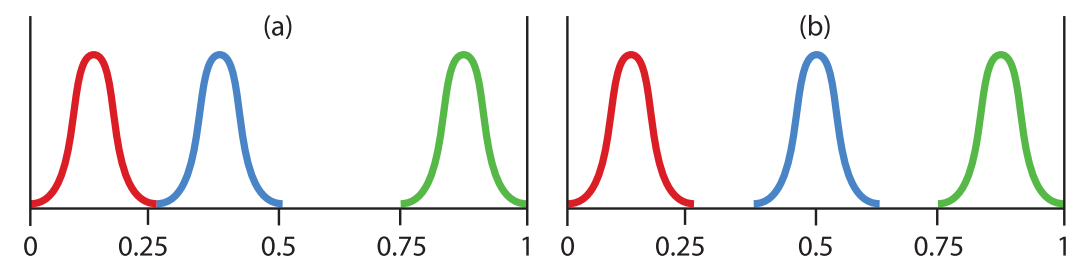

Figure 5. Distributions with equal coverage and spread, but in which (b) shows a larger number of empty spaces between occupied areas, indicating greater polarization in the sense of regionalization. 
Although coverage, spread, and regionalization have appropriate and useful applications, they may be the weakest senses of polarization for many practical reasons:

1. Many surveys report at least one response for each value;

2. They fail to capture the locations of the attitudes;

3. They fail to capture the frequency of attitudes along the spectrum; and

4. Multiple, vastly different distributions will be produce identical measurements.

Although a combination of all three does provide a rough picture, the location and frequency of attitudes matters a great deal in capturing our intuitive notion of polarization. While the refinements below add these features, the simple measures above are distinct features of distributions that should be distinguished in analyses of polarization phenomena. They are most useful for sparse data because they can still achieve exact, high-confidence results in such circumstances.

\subsection{Community fragmentation}

The core of community fragmentation is the degree to which the population can be broken into subpopulations. As a conceptually independent sense of polarization, the more groups there are, the greater the polarization. The number of groups, in and of itself, does not reflect the distribution of attitudes; that is, holding all descriptions of the size and shape of the distribution constant, more separate groups imply more polarization in this sense. Note that in some previous definitions of polarization, a large number of groups indicated a lack of polarization (e.g., Esteban \& Ray, 1994), but their reasoning for such a relationship invoked senses of polarization we have separated from just the consideration of the number of groups alone. The implications this has on a situation depends on what is meant by "group" in that context. Here we consider four different ways of understanding groups.

The first way to think about groups is for when there is no information about the social structure underlying an attitude distribution. Groups can be identified directly from the distribution (histogram) as collections of individuals included within the basin of attraction around peaks as in Figures 6 (a) and (b). In this way the groups are identified endogenously by the patterns in attitude values. This approach identifies and counts the modes in the distribution to determine if it is unimodal, bimodal, trimodal, and so forth (Downey \& Huffman, 2001). ${ }^{3}$ For example, in DiMaggio's figure depicting the attitudes toward abortion (Figure 1 in the introduction) we can visibly discern a group on the far liberal (left) side, and another group in the central position;

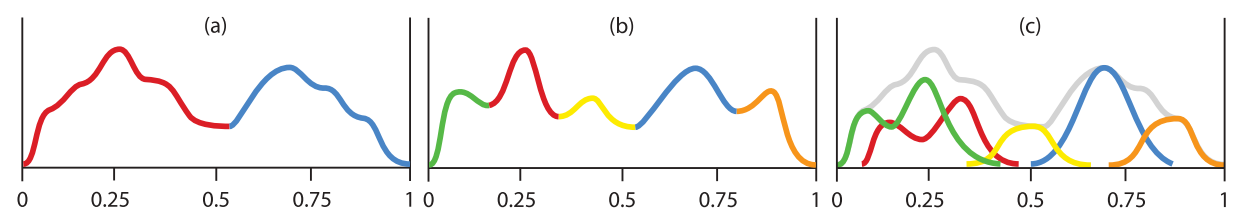

Figure 6. Polarization increases from (a) to (b) for endogenously defined groups. For exogenously defined groups the histogram for the entire population may be broken in to varying numbers of overlapping sub-populations, as in case (c).

\footnotetext{
${ }^{3}$ Our approach here is a naive approach that simply calculates the number of modes as one plus the number of local minima. In the data analysis presented in Section 6 we find cases in which the peak and valley differ only by a few responses, but this is sufficient to reveal an additional group. Such cases may be better captured with a measure that puts minimal variation restrictions on the distribution and/or counts partial groups based on the variation (see Section 5 for more ideas on combined and hybrid measures). Another alternative is to find endogenous groups according to halo-like technique that connects bins together into groups as long as they are sufficiently similar (Mas, Flache, \& Helbing, 2010).
} 
furthermore, in most years there is a third group created by an upturn on the far conservative (right) side. So in this strict sense that data is trimodal and would result in a measure of three groups for most years. We revisit this data in detail in Section 6.3.

Identifying groups in this way precludes the subpopulations from overlapping because group boundaries are defined by the local minima in the distributions. There are several techniques to identify the local minima in discrete data sets; though considerations such as smoothing roughness, bin locations and sizes, and boundary effects complicate these techniques beyond the scope of this article. For our application of this technique to GSS and other survey data in Section 6, we find the local minima bins as those in which both neighboring bins have greater values (or there are multiple bins of equal value forming a flat valley). Half of the contents of minima bins are distributed to each of the groups of which it is a border (for calculating group sizes). ${ }^{4}$ The level of community fracturing is one plus the number of minima, that is, the number of endogenously defined groups.

The second way to define groups is by exogenous variables. In many cases the data are organized by ethnicity, nationality, sex, or other categories, for example, attitudes on education spending grouped by education attainment level. Such an application can create overlapping subpopulations such as in Figure 6 (c). In our data analysis section, we demonstrate both endogenous groups and groups defined exogenously by religiosity.

A third way that appears in the literature is when the data themselves reflect an implicit group membership. For example, for the abortion opinion data in Figure 1 (and much more like it), authors divide the population at the center line into liberal (left of center) and conservative (right of center) responses. The center line may cut the distribution at a valley or peak or anyplace else, and so these user-defined groups will not generally correspond to the endogenously defined groups. Furthermore, because this ad hoc way of distinguishing groups is data-specific and userdefined, it always picks out the same number of groups (so it is not a measure of the fragmentation). This is an approach to define groups exogenously without requiring data beyond the single distribution of responses by making context-based assumptions about the structure of the underlying population.

A fourth way to identify groups comes from agents on networks, grids, or spatial arrangements. Social, physical, and property relationships can all be the basis for grouping individuals together. Furthermore, many of these scenarios allow for degrees of group membership resulting in noninteger numbers of groups and partial group membership. Measures for polarization on spatial and network data require different formalisms even for the same senses presented here. ${ }^{5}$ This topic is left for future work.

Exogenously defined groups provide a way to include categorical data into our analysis framework as long as it is paired with at least one property that holds ordered values along a spectrum. Each category specifies a group, and the subpopulations are plotted together on the spectrum according to the variable with a distribution of cardinal values. For example, in the GSS data presented in Figure 1 we can identify groups endogenously by the shape of the distribution as previously described, or we could group the responses into party self-identification (e.g., Democrat, Republican, other; see Christen et al., 2013). Or we could do both and compare the results (as we do in Section 6). It is important for this sense as a measure of polarization that the number of groups is not known a priori because that is the feature being measured. It is therefore clear that although there are changes in the number of exogenous groups across time and locations, it is the endogenous sense that is more useful as a measure.

\footnotetext{
${ }^{4}$ In applications to higher-dimensional data, endogenously defining the groups requires finding the manifolds of the distributiona well-defined, but much more complicated, similar procedure.

${ }^{5}$ Even in the case of measuring the number of groups, the task is more complicated for network and spatial data. Take, for example, the various algorithms for detecting community structure (and hence the number of communities) in networks. Those network connections only reflect part of the story, and a measure of polarization on networks must combine the community structure of the agents with their attitude values into a single polarization measure.
} 


\subsection{Role of groups in some senses of polarization}

There are other ways to identify groups (such as hierarchical clustering), and some other feature of the population besides what we have called groups could operate as a measure of community fragmentation. Even when the groups are defined in the way presented above, our current project aims at analyzing the polarization in the distributions of attitudes rather than the groups themselves. While the number of groups acts as a distinct sense of polarization, the group identification methods described above also play a role in the measures that follow. Because the measures below require comparisons among subpopulations or sub-distributions, different group identification methods yield variants of the interpretations and polarizations measures to come. When appropriate, one could use different techniques for measuring community fracturing versus the identification of groups for the other senses.

\subsection{Distinctness}

After we have identified or defined groups, we can ask how different these subpopulations or subdistributions are. What matters for polarization in the sense of distinctness is how well we can distinguish the groups. The more clearly the groups can be seen as separate, the more polarized the total population is, regardless of the distance between groups, their size, and the levels of internal consensus (other senses described below). Another way to think about this sense is how unlikely it would be to confuse a person belonging to one group as belonging to another one; the less likely that confusion, the more distinct (and hence polarized) the groups.

This sense of polarization can be captured in at least a few ways. One way is to rank the groups in order of their mean attitude values and then perform pair-wise comparisons of the distributions using the Kolgomorov-Smirnov (KS) two-sample test (Kaner, Mohanty, \& Lyons, 1980; Wilcox, 1997). The KS test is a nonparametric way to examine two sets of data and determines the probability that they were drawn from the same distribution, without making any assumptions about what those distributions might be. The resulting p-values for their being separate distributions act as measures of the distinctness of the groups. A related N-sample test or Bayesian method could potentially extend that approach for any number of groups instead of aggregating pairwise results.

In many applications a simpler measure may suffice to capture the level of distinctness. If the groups are exogenously defined then the overlap between groups (the number of individuals in group $A$ that match attitude values with individuals in group $B$ ) works as a pairwise comparison. The Jaccard distance measure captures this as $1-|A \cap B| /|A \cup B|$. The Bhattacharyya distance measures the dissimilarity between two probability distributions and could also be adapted to our current purposes: $\ln -\sum_{r \in R} \sqrt{y A(r) \cdot y B(r)}$. A less elegant measure of the exogenous distinctness of two groups (but one that is useful for our intended data from surveys and agent-based models) is provided by:

$$
\frac{1}{|A|+|B|} 1-\sum_{r \in R} \min \left\{y_{A}(r), y_{B}(r)\right\}
$$

This formula determines the proportion of the overlap of two distributions by normalizing the sum of the values at each bin of whichever distribution has a lower value in that bin. ${ }^{6}$ When there are more than two groups in the analysis, some aggregation of the pairwise comparisons must be made that also keeps the measure insensitive to the number of groups and the group size disparities. In our applications in Section 6 we add all the pairwise scores and then divide by the number of pairwise comparisons: $\left(G^{2}-G\right) / 2$.

\footnotetext{
${ }^{6}$ To visualize how this works, look at Figure 7 (a) and trace along the minimum of the two distributions from left to right. The sum of those heights will be the area of the gray region, which is then normalized by the total population (the size of $A$ plus the size of $B$ ).
} 


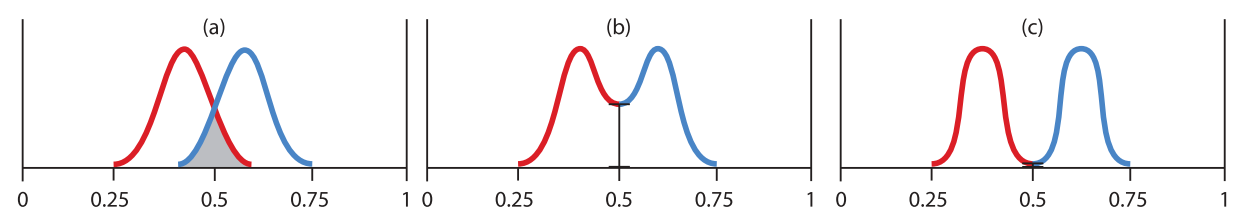

Figure 7. Distinctness scenarios of exogenously defined groups (a) and endogenously identified groups (b). In both cases the distribution in (c) exhibits greater polarization in the sense of distinctness.

As defined in Section 3.5, borders for endogenously identified groups occur at the local minima in the distribution. The greater proportion of individuals that exist at this boundary-the ones that are between the two neighboring groups-the less distinct the groups are. That is, a high frequency of inbetween agents (a mountain pass) is less distinguishing than a low frequency (deep valley). Endogenous distinctness among any number of revealed groups can be calculated via the following formula:

$$
1-\sum_{r \in R} \begin{cases}y(r) & y(r)<y(r-1) \& y(r)<y(r+1) \\ 0 & \text { otherwise }\end{cases}
$$

For endogenously defined groups, the number of group boundaries is $G-1$, so we normalize by dividing by this number to take out the effect of a greater number of groups. In application one must also account for the complication that there may be wide valleys (i.e., several contiguous empty bins) such that multiple bins need to be included in the "in-between" zone. Since some flat areas may be plateaus or outcroppings from a slope, accounting for this detail in identifying only ranges of minima in a simple formula is not possible. Programing a check for these caveats is, however, not at all problematic. Both exogenous and endogenous distinctness are illustrated in Figure 7.

What DiMaggio et al. call "bimodality" is similar to this concept of polarization: people are polarized in this sense "insofar as people with different positions on an issue cluster into separate camps, with locations between the two modal positions sparsely occupied" (DiMaggio et al., 1996). They note that "No issue represents contemporary social conflict as vividly as does abortion, the struggle over which has become symbolic of the so-called culture wars Americans have become more divided in their attitudes towards abortion..." Although we will later see that the trend is not strong, it is the case that attitudes toward abortion between 1970 and 1990 (shown in Figure 1) reflect high levels of polarization in the sense of distinctness because few individuals hold positions between the two large groups.

Bartels' analysis of voting behavior and party identification from 1952 to 1996 reveals that the strength and importance of party identification for voting behavior dropped in the 1960s and 1970s and has rebounded through the 1980s and 1990s. Since their lowest point in 1976, the proportion of "strong" identifiers has increased while the proportion of "weak" identifiers decreased (although these trends are not monotonic). Furthermore, the proportion of voters that identify as "pure" independents has reduced while the independent "leaners" have increased (Bartels, 2000). If we assume, as most do, that the two major U.S. political parties represent positions toward the left and right sides of a spectrum, and that (at least some of) the independents are between these parties, then we can interpret the described change as increased polarization in the sense of distinctness. The frequency of individuals at the locations of the parties has increased while the frequency of individuals between them has decreased. Bartels' information about "strong" and "weak" identifiers and "pure" and "leaning" independents suffices for this conclusion based on the conceptual breakdown being offered, but because he does not provide numbers for which party they identify with it is insufficient to precisely measure this dispersion.

\subsection{Group divergence}

While group distinctness captures how different groups are with respect to being separated (regardless of how far away those groups are in their attitudes), group divergence captures the 
reverse: how distant the groups' attitudes are without accounting for their shapes. The more the groups' attributes diverge the greater the polarization.

For our current purposes it is sufficient to use the normalized distances among the group means (or the area of the convex hull of the means for more than two groups in more than two dimensions). This measure is applicable whether the groups are defined endogenously or exogenously. When there are more than two groups we normalize by the maximum sum of distances possible for $G$ groups on the interval $(0,1)$ (i.e., when all the groups are located at the extremes near 0 and 1$)$. For a set of $G$ attitude subdistributions or subpopulations $\left\{g_{1} \ldots g_{\mathrm{G}}\right\}$, the normalized pairwise differences in group means is:

$$
\text { divergence }=\frac{2}{G^{2}-G} \sum_{i<j}^{G}\left|\bar{g}_{i}-\bar{g}_{j}\right| .
$$

Increasing divergence can be easily imagined as two groups moving away from each other. However, any change that increases the distance of the means will increase divergence, which can also be accomplished while holding spread, coverage, distinctness, etc. constant (as depicted in Figure 8). Thus divergence captures yet another distinct and independent, though common, notion of polarization.

Diverging means is an obvious and therefore a common way to measure polarization. The examples above from both DiMaggio et al. and Bartels for distinctness also slide into divergence. That is, because these measures were not as finely defined in their work, their analysis fails to separate the distinct senses. Our measure tracks the aggregate distances among group means at each time slice, which can then be compared across variables or time. Other researches have examined the interaction terms of the group means and the year (Page \&, Shapiro 1992). That and other versions are explicitly temporal measures with more restrictions, but they are conceptually the same.
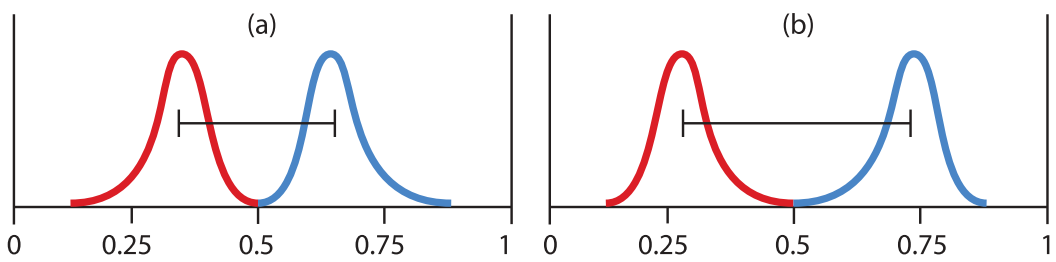

Figure 8. Attitude distribution (b) shows greater polarization than (a) in the sense of group divergence.

\subsection{Group consensus}

Polarization sometimes describes features of a whole population and/or differences among the groups within that population; instead of these, polarization in the sense of group consensus reflects the make-up of the groups themselves. Groups that are more unified in their positions are internally less polarized for the same reasons described in Section 3.2 on dispersion. However, when there are multiple groups, and each group is unified in its attitude, then the society is more polarized. Separate groups that are individually more diffuse already admit to a variety of attitudes, so the less consensus held by each group the lower the polarization.

Group consensus for polarization can be measured via any of the previously considered measures of population dispersion: aggregated in-group absolute deviations, variances, pairwise distances, or even aggregated group spreads. For a set of $G$ attitude sub-distributions (groups) $\left\{g_{1} \ldots g_{G}\right\}$ with population values $\left\{n_{1} \ldots n_{G}\right\}$, our example aggregated group consensus measure is averaged deviations from the group means:

$$
\text { group consensus }=1-\frac{1}{G} \sum_{i=1}^{G} \frac{2}{n_{i}} \sum_{j=1}^{n_{i}}\left|\bar{x}_{j}-\bar{X}_{i}\right| .
$$


As the population of each group becomes more focused on a few attitudes, the closer the average distance to the mean is within each group, and the more polarized the population (as illustrated in Figure 9). This holds whether it is the same set of attitudes for both groups or for other areas along the spectrum. Again recall that this sense is independent of the other senses, so group consensus may increase even as the coverage, population dispersion, distinctness, fragmentation, etc. are all held constant.
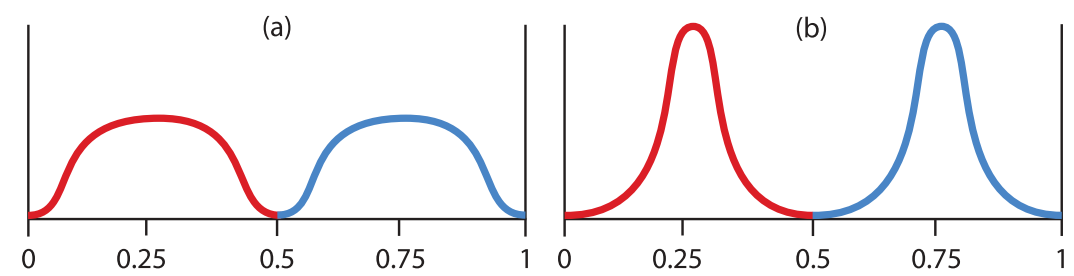

Figure 9. Distribution (b) shows greater polarization than (a) in the sense of group consensus.

We have already noted in Section 3.6 that based on Bartels's analysis distinctness increased in the U.S. voting population from the 1970s to the late 1990s. That is also the case with Congressional votes in the US. In the early 1970s, both Senators and Congressmen voted along party lines about $65 \%$ of the time; in the early 2000 s, that had increased to over $85 \%$ (McCarty et al., 2008). That fact itself does not imply that the two parties overlap less on the issue space, or that they are moving further apart, but it does imply that individuals within the groups are moving closer to the center of their respective groups. So these data support a claim that it is in the sense of group consensus, rather than other senses, that polarization is increasing. Finding that the mean positions of the parties has not significantly changed does not countervail this claim of polarization because they are picking up different features of the distribution.

Baldassarri and Gelman further note increased consensus among voters in terms of particular issue domains. "Looking separately at trends among Republican and Democratic voters we find clear evidence of increasing constraint within issue domains, especially among Republicans. In fact, Republicans have become more consistent on economic and civil rights issues, while Democrats have lost constraint on these issues and become a bit more coherent in their moral views. In both groups of voters, the constraint is growing faster than in the populace as a whole" (Baldassarri \& Gelman, 2008). Clearly these require a multidimensional issue space and a measure of consensus that aggregates for each each party how they have changed across all the issues. We do not provide that here, but it underlines the need for extending these concepts to measures for higher dimensions because it is clear that this feature will not be picked out and distinguishable from other forms of polarization by approaches using correlation and mean squared errors across time and issues.

\subsection{Size parity}

So far none of our senses is directly affected by the sizes of the groups in a population. Our formal measures up to this point either ignore groups completely or focus on in-group aggregates and
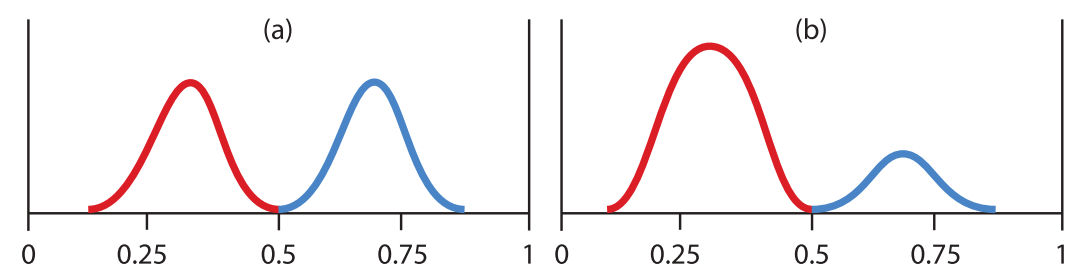

Figure 10. Groups with comparable sizes are more polarized than a large group with smaller outlier groups; therefore in the sense of size parity, attitude distribution (a) shows a more polarized population than (b). 
comparisons of group means. However, it is clear that subpopulation sizes should play a role in the full collection of polarization concepts. Holding the number of groups constant, a society that has one dominant attitude group with a few small minority outliers is less polarized than one with all similarly sized groups (see Figure 10). Although this sense is independent of the particular attitude distributions of the groups, the reasoning here is that (ceteris paribus) a society is more polarized when differing attitudes are held by large segments of the population rather than just small fringe groups.

To measure size parity independently of the other senses requires a formalism that is sensitive to the sizes of the groups while being simultaneously insensitive to the number of groups. A normalized entropy measure fits this description exactly, although for endogenously defined groups this measure may be erratic when the groups change and/or shift boundary bins. For a set of $G$ groups with population proportions $\left\{p_{1} \ldots p_{G}\right\}$,

$$
\text { size parity }_{\text {entropy }}=-\frac{1}{\ln G} \sum_{i=1}^{G} p_{i} \ln p_{i} .
$$

As a revealing application of this measure, consider Americans' opinions regarding the role of women (Bolzendahl \& Meyers, 2004). We ran our polarization analysis on data from the American National Election Studies from 1972 to 2008 reporting individuals' responses (scored from 1 to 7 ) to the following question: "Some people feel that women should have an equal role with men in running business, industry and government. Others feel that women's place is in the home. Where would you place yourself on this scale or haven't you thought much about this?" (ANES, 2010). Many of our measures reveal little change in polarization on this topic even while the mean attitude has moved steadily from 3.92 in 1972 to 1.98 in 2008 . The size parity measure, however, decreased from 0.96 to 0.67 as the population moved from three distinct and substantial clumps toward a distribution that spikes on a score of 1 (equal roles) and falls off quickly as scores increase. In this sense (and in the sense of population dispersion) the polarization regarding women's rights has significantly decreased-society has both moved to greater equality and become more unified (less polarized) on this position.

\section{Measure independence}

The distinct senses above are demonstrably pairwise independent within the constraints of certain boundary conditions: for any two senses one can construct an example from two distributions showing that an increase in one sense of polarization does not require or imply an increase (and could be matched with a decrease) in the other sense. Throughout the exposition we have pointed out this sort of independence and constructed our figures to demonstrate a change in only the specific measure being described. The concepts (and their measures) are not, however, completely orthogonal. If one sets and holds constant the value of a third measure it may force the first two to become positively or negatively related (depending on the particular measures involved). For clarity we now provide an example of such a case.

Recall that a suggested measure for spread is simply the distance between the maximum and minimum observed value. Coverage is the proportion of bins in which at least one person holds that bin's value. And dispersion is the average distance of data points to the mean. Assume that the distribution is not completely filling the bounded (between 0 and 1) attitude space. ${ }^{7}$ To increase spread without varying coverage, one can (for example) move those data points located at positions greater than the median value to larger valued bins. To increase spread without varying dispersion, one can take occupants from near the edge and move one inward and another outward by the same distance. $^{8}$ However, if one fixes the spread of the distribution, then moving points to increase

\footnotetext{
${ }^{7}$ This is one of the boundary condition caveats: If both spread and coverage were $100 \%$ of a bounded region, then decreasing spread would imply a decrease in coverage, although the reverse is not true.

${ }^{8}$ This is a second boundary condition caveat: If each bin initially only has one occupant then moving occupants to increase spread without changing dispersion may be impossible.
} 
coverage (by filling in intermediate bins) will generate a change in dispersion as well. Similarly, keeping coverage constant while accommodating increasing spread requires increasing dispersion.

Other relationships among multiple measures exist as well. For example, if the number of groups equals the regionalization plus one, then the distinctness is already maximal because the groups have no neighboring or overlapping regions. We do not intend to catalog all these potential relationships because they can be identified and explored (if useful) when they come up in specific applications. We have included this section because we believe that it is scenarios such as these that obfuscate the varied senses of polarization, thus creating confusion in analyzing polarization patterns. Also keep in mind that despite their mathematical linkages the polarization senses detailed here remain conceptually distinct. The fact that they are pairwise measure independent is mostly important for demonstrating them to be separate building blocks rather than for practical considerations.

\section{Measure combinations and comparisons}

Capturing the senses independently is vital for understanding the core conceptual basis of how systems might be polarized. Further noting the connections among the measures in applications is necessary to pierce through the confusion that can occur when applying these senses to data. We can get an even deeper understanding of this family of concepts by developing measures that capture multiple senses at once while still keeping the contributions separated. We do not claim that any one of the above senses fully captures any particular widely used notion of polarization, but rather that these senses constitute minimal building blocks of all such notions. These building blocks can be combined in various ways to capture differing, richer notions of polarization while remaining unambiguous about the features of the distribution being described.

As an example, consider the most common measure of polarization in the political literature: bimodality. The use of bimodality implies that the population can be most usefully broken down into exactly two subpopulations, and that a balanced deviation from a moderate position is what it means to be polarized. Fiorina and Abrams use such a measure for American political opinion by tracing the drop in percent of respondents labeled moderate combined with balanced redistribution to both sides of the moderate position (accumulation to only one side did not count as a polarizing trend to them) (Fiorina et al., 2010).

Increases in the bimodality of a distribution can be broken down into increases in several senses of polarization identified here; that is, it is a combination measure of our building block senses. Community fragmentation clearly plays a role because it is important that there are two distinguishable groups. The distinctness of those groups is also important; Fiorina and Abrams are invoking distinctness when they measure the drop in moderate view holders. Group divergence and spread are also included because these increase when equal numbers of responses move to both sides (but less so when they move disproportionately to one side). Neither group consensus nor size parity are explicitly invoked with bimodality, although if there is too little consensus or size parity then the groups may not be visibly discernible as such.

Bimodality aggregates at least three (and up to six) distinct senses of polarization, but the problem with this combination is that, while looking at the same data series, one author can claim that bimodality is increasing while another claims it is decreasing. For example, the two groups could become more divergent (increasing average distance to the mean) while the distinctness (lack of individuals at moderate positions) is decreasing. By separating our measures more finely, we can identify both of these changes and focus our discussion on the substantive importance of both trends rather than a semantic disagreement over whether there is polarization and its appropriate measurement.

Bimodality is not the only extant polarization measure that can be easily broken down. In Axelrod's well-known model of the dissemination of culture, polarization is characterized by the appearance of regions which are internally homogeneous and have no attributes in common with the other internally homogeneous regions (Axelrod, 1997). In this case the groups are endogenously defined, and the more groups the greater the polarization. But it also requires group consensus and distinctness of the agents' traits. Axelrod's meaning of 'polarization' nicely breaks down into the 
concepts offered in this paper even though the traits of the agents are not cardinal values and therefore other measures would be required to quantify some aspects of the polarization. As it turns out, due to the specifics of Axelrod's model, coverage as defined above succeeds in capturing almost exactly the quantity that he identifies even though his description evokes the other concepts. ${ }^{9}$

Another example of a combined measure is the notion of polarization used by Estaban and Ray: "Suppose that the population is grouped into significantly sized 'clusters,' such that each cluster is very 'similar' in terms of the attributes of its members, but different clusters have members with very 'dissimilar' attributes. In that case we would say that the society is 'polarized'" (Esteban \& Ray, 1994). This version of polarization combines (at least) three senses in our breakdown: (1) there needs to be at least two discernible groups that are both (2) widely divergent and (3) have a strong group consensus. For Estaban and Ray's purpose, of teasing out conditions ripe for social unrest, this combination may be the most appropriate, and seeing it as a combination of features facilitates a more refined analysis and a deeper comparison to other polarization phenomena.

Flache and Macy's definition is similar: “By 'polarization' we mean that a population divides into a small number of factions with high internal consensus and sharp disagreement between them" (Flache \& Macy, 2011). For Flache and Macy, "sharp disagreement" seems to imply distinctness either in place of, or in addition to, divergence combined with the existence of multiple groups with high consensus. These examples further highlight that polarization is a cluster concept; neither divergence nor distinctness is necessary for polarization-no single feature of the nine presented here is necessary, but the features can be combined to form broader definitions as appropriate for the application at hand. Seeing them as different combinations, rather than distinct and incommensurate definitions/measures, ties the the disparate versions of polarization together without prioritizing them.

\subsection{Confidence measures}

A further consideration that is left out of the current analysis is the incorporation of confidence into the polarization measures. As you will see, the analysis below uses the percentage of respondents in each category (answer) as the frequency of that response. In some years the number of respondents is fewer than 700 (in weighted terms), while in other years it is greater than 2,000. We do not take account of this response rate variance in our measures. In calculating the number of endogenously defined groups, for example, a valley with a value of 1.0 between two bins with value 10.0 counts just as much as a valley with a value of 9.9 (although that difference in valley height is picked up with the distinctness measure). A more refined measure of polarization of this type might combine both the number of groups, the size parity, the distinctness, and a frequency-based confidence measure. For our current purposes of demonstrating the value of these conceptual distinctions in measuring polarization, these technical considerations are too far afield. Our example measures here are intended to be indicative of these distinctions rather than recommended best practices for teasing these distinctions out of empirical data.

\section{Data analysis}

Our purpose in this article is to help refine and clarify empirical research on polarization so that the discussion can focus on the interpretive questions and substantive issues. As Hirschl et al. point out in their analysis of religious-political polarization, "this task is both empirical and interpretive because an empirical trend is unimportant in the absence of substantive interpretation" (Hirschl, Booth, Glenna, \& Green, 2012). They also make the case that "the research literature is divided on this question with several investigators finding a positive trend in religious-political polarization since 1980, and others finding no polarization" (Hirschl et al., 2012). Although it is not our aim to support one side or the other on this

\footnotetext{
${ }^{9}$ The discrepancy occurs when multiple "islands" of agents coincidently possess all the same traits values.
} 
debate (or any other debate), we will reevaluate the presence of religious-political polarization through our collection of measures and use these results to reassess the support for their substantial claims.

Although debates that cross over multiple data sets tend to burn hotter, and suffer more from incongruous analysis approaches, we will restrict ourselves in this article to analyses that pull their data from the General Social Survey (GSS) dataset. The GSS contains responses to questions on a broad spectrum of topics, including many that are critical for understanding trends in political and social attitudes. The breadth (> 5,500 variables) and continuity (1972-2012) of the GSS makes it an excellent data source for social scientists.

However, few of the questions have responses keyed on a 1-6 (or higher) Likert scale. Instead they are limited to 5 values or less or have categorical answers; many are true/false or agree/disagree questions. Although the frequencies of responses in binary or categorical data can reveal trends in attitudes with respect to a specific question, addressing polarization in a broader topic (such as women's rights, religiosity, or intellectualism) typically requires inferences from examining patterns in multiple questions. To measure polarization on the GSS data using the example measures presented above requires us to limit our application to those topics with appropriate ranked-order scoring systems. ${ }^{10}$

\subsection{Political polarization}

As a demonstration of our technique to social-political data, we first address the question of whether there has been polarization in the American populace's general political views. We take the normalized frequencies of the responses to the POLVIEWS question of GSS and apply the above measures to the responses for each year the question was reported. Because we are using a single variable here, the groups are endogenously defined and the group-dependent measures are calculated on the revealed subdistributions.

The Hirschl et al. analysis reveals (among other things) that among some Americans (nonsouthern Christian white males) there exists a greater degree of religious belief correlating with a greater likelihood of voting for Republican candidates and a lesser degree of religiosity correlating with a decreasing likelihood of voting Republican. The effect is amplified among the wealthiest quarter of the population. Their analysis includes details that are not important for our discussion here; our goal is not model validation. Instead, we wish to determine to what degree the kind of politico-religious polarization detected by Hirschl et al. can be picked out and measured by our technique.

Rather than looking at specific voting behavior, we investigate the presence of polarization in the more general political views captured by the POLVIEWS question of the GSS. The question is: "We hear a lot of talk these days about liberals and conservatives. I'm going to show you a seven-point scale on which the political views that people might hold are arranged from extremely liberal-point 1 -to extremely conservative-point 7 . Where would you place yourself on this scale?" First we apply our measures to the political views data by itself, and then we apply our measures to the political views data using the BIBLE question (described below) to define exogenous groups. The POLVIEWS data for the first analysis are filtered by those respondents who also answered the BIBLE question so that our analysis of political views here will match up in years and sample size with our analysis below (see Figure 11).

Some measures (such as spread, coverage, and regionalization) are not relevant for this analysis because, as is typical for survey data with a limited number of possible responses, nearly all the bins

\footnotetext{
${ }^{10}$ Specifically, our technique requires ordered response values rather than categorical responses and excludes responses of "no response," "don't know," "can't choose," and the like. Some questions (like POLVIEWS described below) ask participants to rank themselves on a 1-7 scale. These variables can be analyzed without analytical gymnastics across multiple variables. The ABORTION variable we use is, however, a composite of multiple true/false questions that are added together to produce a 0-8 scale.
} 

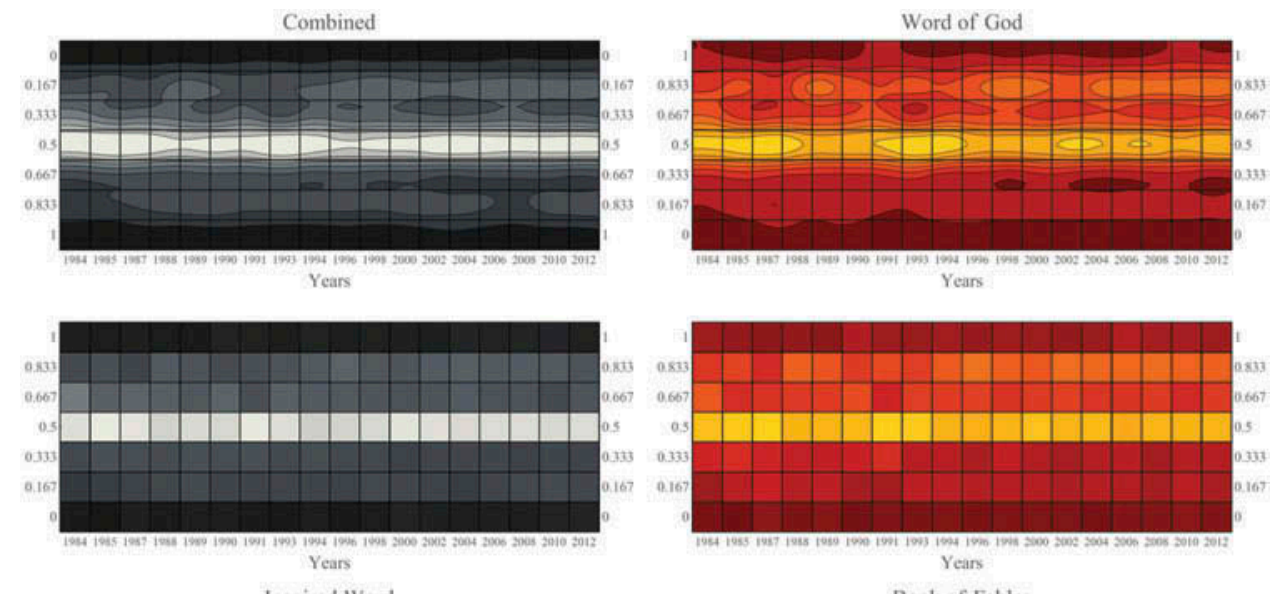

Inspired Word
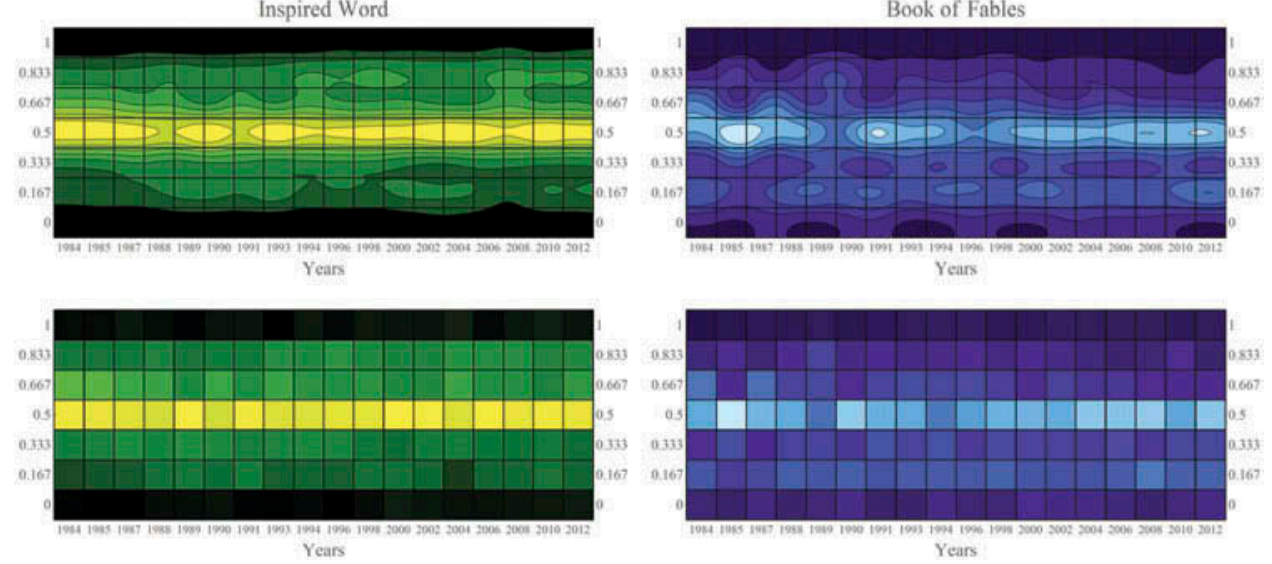

Figure 11. The POLVIEWS data by religiosity category and combined. Contour plots at the top of each pair show the data with interpolation order 2, the heat maps on the bottoms are color codings of the raw GSS data presented in the Appendix.

have at least one entry. ${ }^{11}$ Figure 12 shows the results of the relevant polarization measures to the political views. The two most striking results in this plot are:

1. The population dispersion climbs slightly, but not monotonically, from 1984 to 2012; and

2. The number of endogenously defined groups, as well as the measures across those groups, undergo a striking and lasting shift in 1996 (see Table 4 in Appendix for the actual measure values).

None of the group-based senses of polarization can be measured when there is only one group, and when groups are being defined endogenously the existence of a single group indicates a single-peaked distribution. In order for there to be an increase in the number of endogenously defined groups, it must be that the distribution has shifted to produce more peaks and valleys. What happened in this case is that in 1996 the proportion of respondents who described themselves as strictly conservative increased from $16.3 \%$ to $18.4 \%$, which put that bin above the proportion who described themselves as

\footnotetext{
${ }^{11}$ For the political views data taken together, no bins are empty. When broken down by BIBLE, in 1984 there are no respondents who chose extremely conservative and answered that the bible is a book of fables - and that is the only empty bin. For the abortion analysis below, there are a few scattered empty bins for the book of fables group, likely the result of low sample size in some years. They are left out of the analysis because they do not indicate coherent patterns in the data.
} 


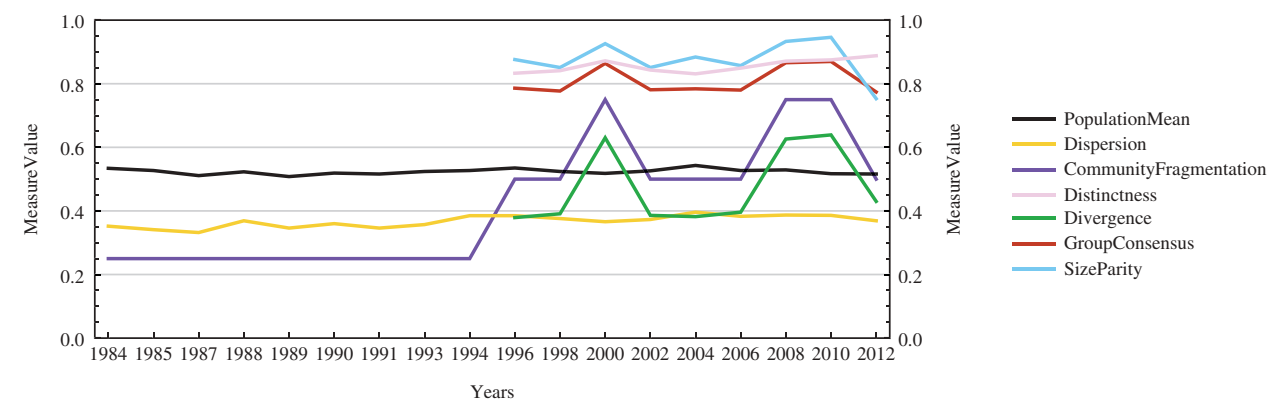

Figure 12. The relevant measures of polarization by year for the POLVIEWS variable filtered by BIBLE.

slightly conservative (which went from $16.6 \%$ to $16.7 \%$ ) creating a new peak at the conservative bin. This increased the number of groups, a measure of the community fracturing sense of polarization, but half the bump in conservative can be attributed to an influx from the extremely conservative bin (from $3.9 \%$ to $2.8 \%$ ). The increase in polarization in this case, therefore, is not an overall move to the right, but rather a pull in from the right that increased focus on the pure conservative bin.

In 2000 the same thing happens on the liberal side: the pure liberals outnumber the slightly liberals to generate a third mode in the population distribution. That doesn't last, and the liberal side is strictly decreasing again until 2008. At that point the trend first creates another group divide at slightly liberal and then the number of groups goes down to two in 2012 because the 14-year conservative peak dissolves. By itself the community fragmentation measure doesn't distinguish where on the spectrum the groups are occurring, only the number of groups. Although there is sometimes two and sometimes three groups after 1996, there is no clear increasing or decreasing pattern. ${ }^{12}$

The other group-based measures are normalized by the number of groups to isolate the individual senses; however, the group composition clearly plays a role in determining these measures. From 1996 to 2010 there is a group divide at slightly conservative, and the size parity jumps when a third group also forms (in 2000, 2008, and 2010). In 2012 the single group divide is at the bin representing slightly liberal, and the conservative and moderates are all in a group together. As one can tell from the higher than $50 \%$ population mean value, the total population leans right, so the size parity plummets when the distribution shifts in this way. Although the direct effect of the number of groups has been distilled from that measure, cutting the population up in different ways can produce sudden, drastic changes in other measures.

More important than any of these specific results is (1) the differences in the polarization for the different measures and (2) the difference between this collection of measures and other approaches. The population dispersion experiences a slow and steady increase despite the abrupt changes in the shape of the distribution picked up by other measures. The suite of measures provided here capture each aspect of that change separately to provide a thorough description of the attitude dynamics. They do not reveal a consistent polarizing trend in Americans' political views. Within the timespan for which groups are observed (1996-2012) group divergence, distinctness, consensus, and size parity do not consistently change, so we have just one sense in which the population polarized: community fragmentation.

This one sense in which we do see polarization is connected to Fiorina and Abrams' notion of bimodality, although more subtle because it is not the case that moderates decrease and the extremes increase. The extreme positions vary, but not in a consistent way. The movement is from nearly moderate positions to slightly less moderate positions on both sides. That sounds similar to what was intended by bimodality, but it would not be picked up by a direct bimodality detector because the percent of moderate responses actually increase through the 1994 to 2000 period in which the

\footnotetext{
${ }^{12} \mathrm{~A}$ regression analysis between the community fragmentation measure and the year naturally reveals a positive relation with high confidence, but with so few samples (18 years) and a single variable this is not an appropriate characterization of coherent patterns in the data.
} 
number of discernible groups increases. Our measure of community fragmentation (essentially the number of modes) picks up lopsided changes, and it is agnostic with regard to the global distribution of attitudes (e.g. generally normal, inverse normal, or fat tailed). It only does one thing, and it does it precisely. Because the overall population is not appreciably drifting apart (as measured by population dispersion and group divergence) one may be inclined to deny the presence of polarization. But those are different senses of polarization than fragmentation, and in the sense of community fragmentation there is an increase in polarization from the pre-1995 period that persists throughout the post-1995 period.

\subsection{Political polarization by religiosity}

Although some authors have pointed to religion as being a strong driver of social polarization (e.g., Evans, 2002; Hunter, 1991), ideas differ regarding how to categorize religiosity. Following Hirschl et al. we adopt responses to the BIBLE question from the GSS as a reasonable proxy, or indicator, of respondent religiosity. This question asks participants "Which of these statements comes closest to describing your feelings about the Bible?" Respondents must choose one of the following answers (or opt out):

(a) The Bible is the actual word of God and is to be taken literally, word for word.

(b) The Bible is the inspired word of God but not everything in it should be taken literally, word for word.

(c) The Bible is an ancient book of fables, legends, history, and moral precepts recorded by men.

Because there are only three responses available for this question it is not recommended that one apply our measures to this attitude distribution directly. Instead, we use these three responses to define exogenous groups, and use these groups to run our polarization analysis on the same political views spectrum above. For the BIBLE question, there are no empty bins for any years, so the community fragmentation measure is entirely determined by the number of possible responses to that question-in this case three. ${ }^{13}$

It is not surprising to find that the mean political views of the Word of God group is consistently more conservative than the Book of Fables group. Furthermore, except in 1997 when the Word of God groups scored slightly less conservative than the Inspired Word group, the more religious groups were also increasingly conservative from 1984 to 2012. It is clear from Figure 13 that the population dispersion and the average in-group dispersion values captured by group consensus are nearly identical. Group consensus is higher because the largest group, Inspired Word, has a lower dispersion than the other two (which are nearly identical), and the group consensus measure averages the three groups without adjusting for their sizes.

None of the measures show high degrees of fluctuation or dramatic trends, so any claim to polarization of political views by religiosity must be weak. The clearest sign of increased polarization is from group divergence (the average distance among the group means). The value in 1984 is 0.1170 , and the value in 2012 is 0.1774 ; although the rise is not monotonic, it is the case that only two years prior to 1996 reached above 0.12 , while every year after 1996 scored above 0.12 . Hence there is some indication that the average political views of the highly religious and secular groups are crawling away from each other.

Size parity also shows a slight trend of increasing polarization: it rose from the lowest value (in 2002) of 0.8752 to 0.9589 in 2012 . This reflects a sharp increase in the percentage of respondents who gave the Book of Fables answer from $14 \%$ to $22 \%$, most of which came from the largest group-

\footnotetext{
${ }^{13}$ There are cases, for other questions, in which some response values are not given by anybody for some years. If these questions are used to exogenously define groups, then the number of groups will change across time. As mentioned earlier, this is rare for survey data, but when analyzing simulation models it is common for predefined types of agents to enter and leave the population.
} 


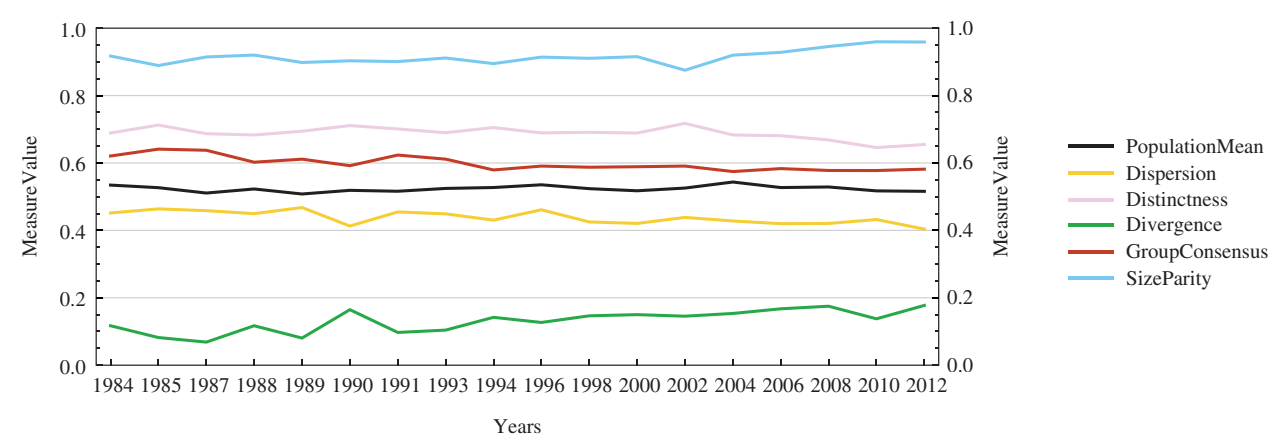

Figure 13. The relevant measures of polarization by year for the POLVIEWS variable grouped by BIBLE.

Inspired Words went from $56 \%$ to $46 \%$. These religiosity group size changes do produce changes in the political view distributions, so the do not show up in the other measures. That is, changes in size parity are measures of the polarization of political views through changes in religiosity rather than changes in political views. ${ }^{14}$ However, since the lowest value of size parity occurs in 2002 we cannot make the claim that there is an overall increasing trend, only that the sizes of the religiosity groups have been equalizing over the last 10 years of data. ${ }^{15}$

The measures of distinctness and group consensus have negative trends, but only barely. For distinctness there is a negligible increase from 1984 until the high point in 2002, after which it declines noticeably. Distinctness went from a high of 0.7175 in 2002 down to 0.6548 in 2012, meaning that there was an $8.68 \%$ increase in the average amount of overlapping responses among the groups. The religiously separate groups are becoming politically less polarized with respect to their distinctness. Group consensus reveals a more consistently downward trend, but the difference in values is too small to make any conclusions.

All together our measures indicate that the political polarization of the populace (1) is minor, (2) is confined to specific aspects of the distribution, and (3) is increasing or decreasing depending on the aspect examined. Furthermore, although the supposed religious divide certainly exists and is reflected in the groups' political views, it is only slowly widening. That widening may be offset by the flattening of political attitudes within the religious groups, and perhaps a disconnecting of religious and political views.

\subsection{Abortion rights polarization}

We now turn our attention to an issue that, while not directly political, is politically heated and tied to religiosity: abortion rights. The abortion rights issue has drawn a lot of attention because it is a hot media item and because the data so clearly reveal different modes of thought on the issue (recall Figure 1). Previous analyses have remarked that the attitude distribution on this topic is divided but stable (Bolzendahl \& Meyers, 2004; DiMaggio et al., 1996). We already referenced abortion opinion data in the introduction and to demonstrate endogenous group distinctions in Section 3.5; now we present a full polarization analysis of the GSS abortion data (see Figure 14). The role of religiosity in explaining the split in opinions has been addressed by Evans (2002) and others, but their methodologies are not directly comparable.

\footnotetext{
${ }^{14}$ Because the GSS data used here are not from longitudinal studies, we are not detecting individuals changing their religiosity, but just a reflection of changes in the cross section of social attitudes.

${ }^{15}$ One must resist the inclination of assigning a negative connotation to polarization in the interpretation of these results. Here polarization is a purely descriptive term. It may very well be beneficial to have equal numbers of people in each group in some cases, and everybody in one group for other cases. The context determines whether high or low polarization values are desirable.
} 

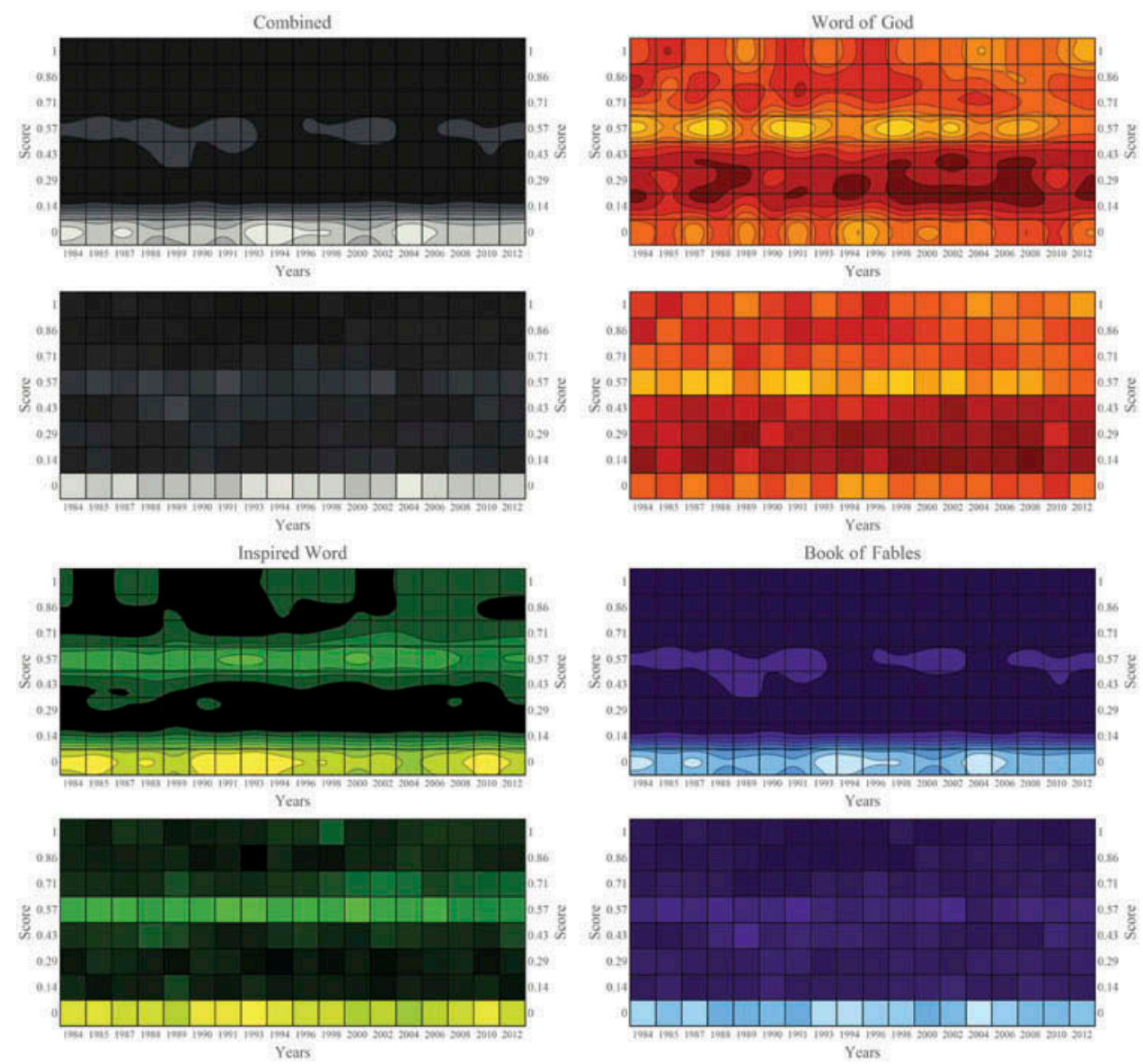

Figure 14. The ABORTION data itself and broken down by by religiosity category. Contour plots at the top of each pair show the data with interpolation order 2, the heat maps on the bottoms are color codings of the proportions in the raw GSS data presented in the appendix.

The GSS has included a variety of questions regarding opinions about abortion issues that varied by year. Overall opinions about abortion are determined by aggregating the responses to these questions to produce a fuller time series dataset (with respect to numbers and years of responses). ${ }^{16}$ The results of applying our measures to the abortion opinions data are presented in Figure 15. Just as before, the values for spread, regionalization, and coverage are left out because, as is typical with Likert scale survey data, all bins are occupied yielding values of one, zero, and one respectively.

With eight possible responses, it is still the case that the maximum number of endogenously defined groups is four. The actual number of mode-defined groups is consistently three (except in 1990 when there are four and in 2008 when there are two), but the locations of the minima that separate these groups constantly change locations. First note that when strictly measured, the number of modes in the data is only equal to two in a single year: strictly, speaking, the data are not bimodal. There are two dominant peaks, but the distribution is typically trimodal.

\footnotetext{
${ }^{16}$ Specifically, the ABORTION variable used here is aggregated by COMPUTE from the study /html/D3/GSS12 and reported by the SDA tool (Thomas, 2013) as the sum of ABDEFECT, ABNOMORE, ABHLTH, ABPOOR, ABRAPE, ABSINGLE, and ABANY (Smith, Marsden, Hout, \& Kim, 2013). First we analyze the ABORTION variable by year filtered by BIBLE, and then grouped exogenously by BIBLE (paralleling our analysis of POLVIEWS above). The composite ABORTION variable has response positions ordered from more liberal to more restrictive, whereas the POLVIEWS had seven.
} 


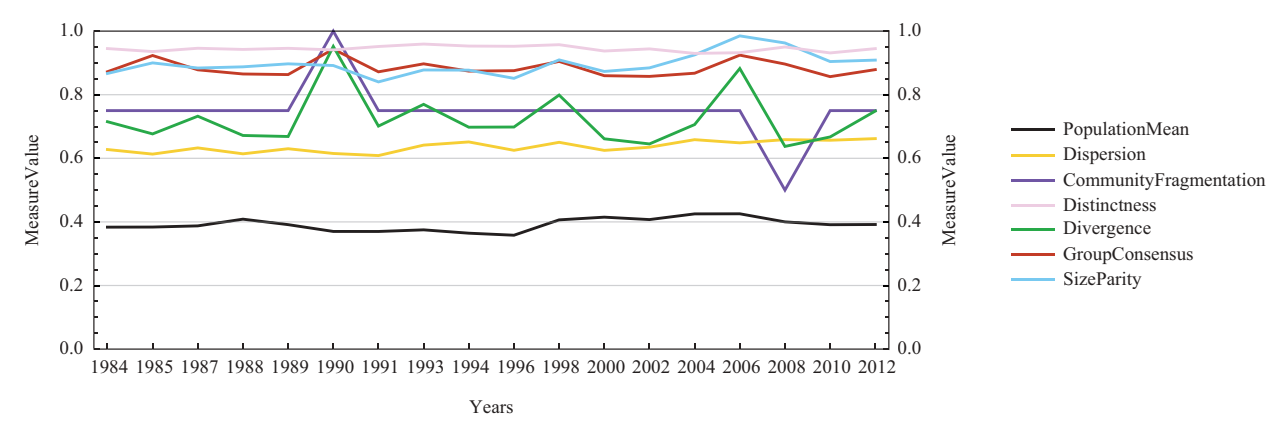

Figure 15. The relevant measures of polarization by year for the ABORTION variable filtered by BIBLE.

As a result of the moving valleys, the responses included in each group are constantly shifting, and this produces volatile group-based measure values. Volatile though they may be, there is no clear trend of increasing or decreasing polarization (whether taken as a comparison of initial and final values, rolling averages, or pre/post 1996 values).

A closer look at the dynamics reveals some further patterns. As shown in Figure 15 the distinctness measure's lowest value is 0.9299 -meaning that at most $7 \%$ of the responses occur in boundary bins. This holds true even as the locations of boundary bins move. This measure therefore characterizes the deep troughs apparent DiMaggio's figure; the attitude space between groups is very sparsely populated.

That figure, however, misrepresents the seeming size disparity between the attitude groups. The liberal side is a single bin with roughly $35 \%$ of the population. The group at the center position looks much shorter, but it spans several bins. These responses all together constitute a flatter group of slightly greater size. The shifting third group (which is sometimes between the two dominant groups and sometimes at the far conservative side) is always small (typically less than 10\%), but the size parity measure still consistently scores around $90 \%$. Note that if we forced there to be two groups, liberals and conservatives, then this would heavily distort the measures of groups sizes and their similarity.

The divergence measure shows the greatest amount of fluctuation which reflects the changes in where that third group is located. A third group on far right obviously produces greater divergences measure values than a group located between the two big ones. The population dispersion does show a slight increasing trend, although the magnitude of the increase is too small to make any claims of significance. And although it seems that the less liberal group is becoming flatter over time, the group constitution changes too much for the group consensus measure to track it. The result is group consensus that fluctuates with the group constitution but produces no clear trends.

The overwhelming conclusion is that there is no substantial increase or decrease in polarization levels in any sense with respect to American's views towards abortion rights. Although there are changes from year to year, none of them produce discernible trends. Our more nuanced analysis of the separation of ideas reveals a consistent and persistent division of views, but not one that is changing coherently over time.

\subsection{Abortion rights polarization by religiosity}

Now we move on to an analysis of abortion by the same three categories of religiosity that we used to analyze political views (see Figure 16). Naturally the population dispersion is unchanged, and the group size parity is the same as in the POLVIEWS analysis because the exogenously defined groups are identical. Again there are no stark trends, and only divergence reveals sharply erratic patterns. Recall that our example measure of group divergence is the normalized distance among the group's means. The mean value for the secular Book of Fables group remains nearly constant throughout the 


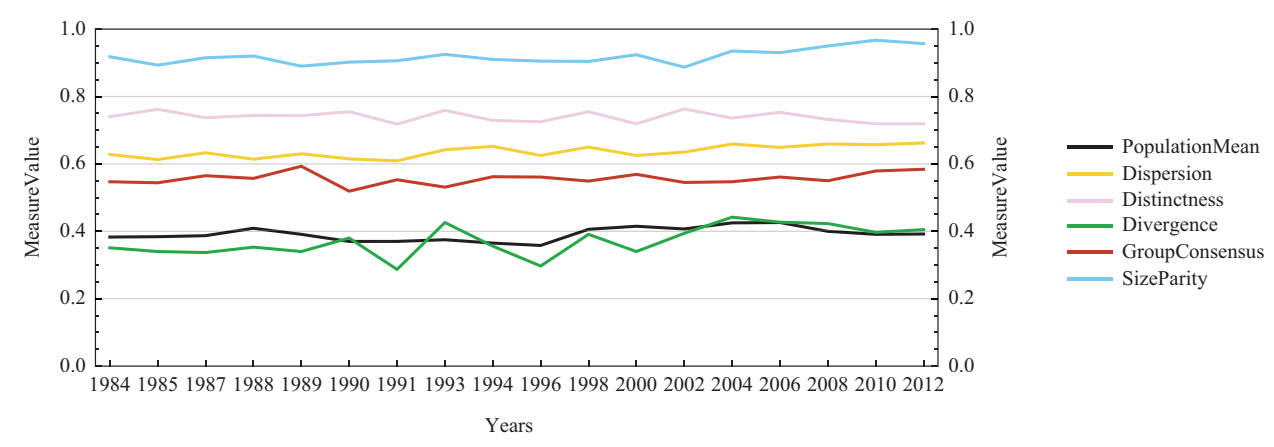

Figure 16. The relevant measures of polarization by year for the ABORTION variable grouped by BIBLE.

whole period. ${ }^{17}$ During this time, however, the Word of God group climbed spasmodically from 0.526 in 1984 to 0.594 in 2012. The Inspired Word group experienced an up-swell between 1994 and 2008 , keeping it roughly in the middle. These changes are revealed as a boost in the group divergence measure for the period after 1996 compared to the earlier years.

Because the groups were moving apart, the divergence increased, but because nearly half the respondents are in the middle group, and the groups significantly overlap, the overall population dispersion increases only slightly over the 28 -year period. The gradual increase only amounts to a $5.85 \%$ change in dispersion, and the increase is not monotonic. Along with that change we see the group sizes becoming more equalized; fewer individuals take it literally and more consider it a book of fables (although the latter remains the smallest group).

When grouped by religiosity, opinions on abortion may be becoming more polarized in the senses that they are getting further apart on average and that the religious group sizes are becoming more similar. The dispersion increase can be attributed entirely to the strongly and moderately religious groups becoming more anti-abortion while not flattening out. None of these polarization measure changes are large because neither public opinion on the topic nor people's religiosity have changed dramatically since 1984 .

\subsection{Comparison of political views and abortion}

As mentioned in Section 2, our comparative measures can be used to detect trends or to evaluate the differences in attitude distributions across topics. To demonstrate this second usage we now compare the measures of attitudes regarding abortion to those of political views across the whole time span. We will also briefly compare the results from the endogenously and exogenously grouped analyses.

For the political views data, the clearest difference between the endogenous and exogenous analysis is community fragmentation. This variable has a constant value of three for the exogenous analysis, but reveals the greatest change in polarization for the endogenous case. This becomes more insightful when one considers that the exogenous analysis reveals that increasing religiosity correlates well with increasingly conservative political views, but that the mean positions of these three groups have been slowly diverging. The endogenous analysis uncovers the formation of distinct lumps in the distribution corresponding to these religiosity groups as they move further away from each other. The relationship between religiosity and conservatism is no surprise, but it is surprising that the separation of the religious groups with respect to their political views sufficiently (though not necessarily) explains the appearance of new modes in the political views distribution. This comes

\footnotetext{
${ }^{17}$ The attitude of this group fluctuates only slightly around its mean value of 0.1819 on a 0 to 1 scale in which higher numbers are more anti-abortion.
} 
out despite indications that the polarization in the senses of group distinctness and size parity are decreasing for the religious groups.

Endogenous comparison: In light of the above connection, comparing the endogenous group community fragmentation between political views and abortion produces a curious result. For political views there was just one mode until 1996, with demarcation lines highly consistent afterward. In contrast, the number of groups for abortion rights was three for all but 1990 and 2008. Also, the intergroup positions for political views were largely consistent even as the number changed, but the demarcation lines were constantly in flux for abortion despite the consistent number of groups. The difference is that American political views tend to be normally distributed, but with fringe groups of varying sizes to both the right and left, while the baseline distribution of abortion opinions is bimodal with varying proportions appearing at the anti-abortion extreme and in the broad valley that separates the two dominant groups. With respect to the community fragmentation sense of polarization, political views have recently become nearly as polarized as people's position on abortion, although the distributions look very different.

The virtue of this fine-grained analysis of distinct senses of polarization is clearer from the comparison of more nuanced senses. Comparing the analyses of political views after the schism in 1996 and abortion in which the groups are endogenously defined, we can see subtler differences than the number of groups reveals. For example, the distinctness of these endogenous groups is consistently very high (near $95 \%$ ) for abortion, but consistently near $86 \%$ for political views. Distinctness measures the percent of responses not in boundary bins, so on average there are $2.5 \%$ of responses in the bins between groups for abortion and between 5 and $7 \%$ for political views. The groups of attitudes about abortion are 2-3 times more distinct than the ones for political views.

Group divergence (normalized distance among group means) is volatile for both datasets: political views toggles between 0.40 and 0.63 depending on the number of groups; while abortion tumbles between 0.637 and 0.952 without being tightly coupled to the number of groups. Thus people's attitudes are consistently more polarized about abortion than political views in the sense of divergence.

The group consensus of political views stays close to the average value of 0.81 across the post1996 years. Abortion opinion group consensus has a slightly wider range around a slightly higher value of 0.88 . Size parity is similar for the two datasets-staying in the high 80 s and low $90 \mathrm{~s}-$ although the political views data are more volatile. The overall result is that across more senses of polarization, people's views toward abortion are more polarized than their political views when groups are defined endogenously.

Exogenous comparison: With groups defined exogenously by the religiosity categories, the subgroup distributions can now overlap. The average distinctness of the groups for abortion opinions is 0.7394 , and for political views it is 0.6886 . So for political views there are $5.11 \%$ more people in overlapping regions of the attitude space. The difference between these values and the endogenous distinctness values indicates the degree to which the religiosity categories explain the modes in the data. Although some more detailed analytics are possible, at this stage we will just point out that the religious groups explain (in a quantitative way) the modes in the distribution of political views better than those found in the abortion data.

The across-time average group divergence for abortion is nearly three times the group divergence for political views $(0.3715$ versus 0.1307$)$, but groups in both data have similar concentrations around their means (average consensus is 0.5564 versus 0.5985 ). Thus the largely normal distribution in political views does not breakdown into clear religious groups, but the distribution of abortion opinions does line up. Putting these three measures together informs us that religiosity explains a small amount of the polarization in people's political views, but it explains a great deal of the polarization in abortion opinions. The difference derives from a combination of multiple features of the distributions and cannot be summarized by a single simple measure.

Although views on abortion are cleanly separated at the population level, when broken down by religious categories they are actually less polarized in the senses of consensus, divergence, and 
distinctness but not of size parity. This pattern also holds for political views. So categorizing by religiosity yields groups that are closer together, more spread out, and share more attitudes than the modes of the data. That is not mathematically implied by the measures, and grouping by a different variable (e.g., ethnicity or education) may reverse one or both of these relationships.

Our more nuanced measures of polarization reveal more nuances in the value and trends of polarization in attitude data. The added detail makes descriptions of the results more complicated. Although rougher and more aggregated measures may make it easier to make definitive claims about polarization in society, they fail to capture the full picture and therefore mis-represent the subtle variations in the distributions of attitudes, and the many independent ways they may change over time.

\section{Conclusions}

A clear understanding of any social phenomena demands a clear set of tools for description and analysis. Here we focused on the polarization of attitudes among individuals along a spectrum. That focus reflects our application interests and a desire to keep the descriptions of the data simple rather than a limit to the applicability of the distinctions. The nine senses presented here apply across disciplines and for any property distribution, whether discrete or continuous, although the particular measures used may require adjustment to best handle particular data sets. Most, and possibly all, of the distinct senses appear in the literature as definitions of polarization, but not the use of an exhaustive battery of measures nor a deep look into how each measure reflects features of the data.

Clearly not all the senses presented in this article are by themselves equally strong matches for an intuitive and inclusive notion of polarization, but each captures a key aspect. The senses are not competing to be the "real polarization"; instead we recognize that polarization is a multifaceted feature of attribute distributions and changes is those attribute distributions. Measures that combine multiple features do have their uses, and we briefly explored them in Section 5. However, popular measures of polarization are not of an unambiguously decomposable type that we suggest. Existing measures of polarization can be decomposed into our senses, but the translation is not unique. Hence we propose that capturing the independent components of polarization is more fruitful for producing consistent assessments and comparable results.

Our goal has been to provide and explicate a suite of definitions for polarization phenomena. As a means to that end we applied them to sociological data on Americans' attitudes regarding their political views and on abortion (and a few others). These two topics were analyzed both with exogenous groups defined by categories of religiosity, and also filtered by that same variable to ensure the set of respondents was identical. The result of our polarization analysis is that there are few clear trends for any segment of the data, and no strong trends of increasing or decreasing polarization for political views or abortion from 1984 to 2012. There are weak trends, but also weak trends of decreasing polarization in some senses. The exaggerated perception of a political divide in the United States may derive in part from an emphasis on issues, like abortion, which do reveal a clean split in attitudes held by groups with other salient differences (e.g., religiosity).

The senses in which polarization is evident are not those commonly detected. For example, the indicated trend for political views is not bimodality (people going out from the center to the extremes), but rather a congregating of attitudes into three camps. The central position has actually gained support as these fringe groups have broken away from the otherwise normally distributed attitudes. A similar observation has been made for congress voting nominate scores: the mean positions of each party have not significantly moved, but there has been a marked increase in group consensus (Gelman \& Palmer, 2011). Our preliminary polarization analysis of the DW-nominate data revealed larger and clearer differences for both endogenous and party-defined exogenous groups. Our follow-up in-depth analysis will determine whether there are more nuanced patterns in the trends and comparisons of our refined senses of polarization. 
In addition to the application to DW-nominate score data, we will pursue an expanded collection of alternative measures for each sense of polarization. All the measures presented here can be refined and adjusted in many ways-too many ways to cover in any one article. We would like to identify (or establish) best practices for the use of these measures that match data characteristics (e.g., number of samples, number of bins) to the best measure(s) for that data. These refinements can be pursued as part of applications of the senses described to other issues and datasets.

Our expansion plans take us into territory requiring more distinct senses. The first of these expansions is into multi-dimensional data. All of the senses and measures here have applications in higher-dimensional spaces, but we are also interested in new senses of polarization that the higher dimensionality makes possible. As already mentioned, the correlation (or association) of ideas along multiple dimensions is an oft-used sense of polarization left out of the current analysis because it requires multiple dimensions. But that is surely not the only such sense. We aim to provide a more expansive catalog of the senses of polarization that depend on greater dimensionality.

In direct connection to our own simulation work, we also plan to extend our senses of polarization to those that exist on networks. The incorporation of a network structure connecting the individuals complicates both the conceptual distinctions and the measurements. For example, although the distribution of attitudes will still have (say) endogenously definable groups with distances between their means, the polarization of the structured society will also depend on whether individuals with similar attitudes are connected to each other in the network or are in different weakly connected network communities. Measures such as the path length among nodes weighted by the similarity of their attitudes indicate the direction we are going, but we are still exploring the full breadth of conceptual distinctions available for variations in attitudes across network structures.

We have started the development of a suite of senses of polarization by putting forward nine independent ways in which polarizing features of distributions can be captured. For each way we have provided at least one example measure and the reasoning for why greater or lesser values are considered to illustrate greater or lesser polarization. There is a bound to how many independent ways a frequency distribution can be captured, and while we are not claiming exhaustiveness, our collection of nine senses is a strong start in providing a full methodology for describing and analyzing polarization in attitudes (aka beliefs, opinions, views, ideas, attributes, etc.) in social contexts. Using our expanded set of senses and precise polarization measures will bring greater clarity to social analysis and allow social scientists to focus on the substantive issues at hand.

\section{References}

ANES. (2010, August). Equal role for women 1972-2008: Table 4c.1. Retrieved from www.electionstudies.org/

Axelrod, R. (1997). The dissemination of culture: A model with local convergence and global polarization. Journal of Conflict Resolution, 41, 203-226. doi:10.1177/0022002797041002001

Baldassarri, D., \& Gelman, A. (2008). Partisans without constraint: Political polarization and trends in American public opinion. American Journal of Sociology, 114, 408-446. doi:10.1086/590649

Bartels, L. M. (2000). Partisanship and voting behavior, 1952-1996. American Journal of Political Science, 44, 35-50. doi:10.2307/2669291

Bolzendahl, C. I., \& Meyers, D. J. (2004). Feminist attitudes and support for gender equality: Opinion change in wornen and men, 1974-1998. Social Forces, 83(2), 759-789. doi:10.1353/sof.2005.0005

Brownstein, R. (2007). The second civil war: How extreme partisanship has paralyzed Washington and polarized America. Penguin Press HC.

Christen, M., Ott, T., \& Schwarz, D. (2013). A new measure for party coherence: Applying a physics-based concept to the Swiss party system. Advances in Complex Systems, 16(6), 1350011. doi:10.1142/S0219525913500112

Dandekar, P., Goel, A., \& Lee, D. T. (2013). Biased assimilation, homophily, and the dynamics of polarization. PNAS Early Edition, 1-6. doi:pnas.1217220110

DiMaggio, P., Evans, J., \& Bryson, B. (1996). Have Americans' social attitudes become more polarized? American Journal of Sociology, 102, 690-755. doi:10.1086/230995

Downey, D. J., \& Huffman, M. L. (2001). Attitudinal polarization and trimodal distributions: Measurement problems and theoretical implications. Social Science Quarterly, 82(3), 494-505. 
Esteban, J., \& Ray, D. (2012). Comparing polarization measures. In Michelle R. Garfinkel and Stergios Skaperdas (Eds.). The Oxford handbook of the economics of peace and conflict. doi:10.1093/oxfordhb/9780195392777.013.0007

Evans, J. H. (2002). Polarization in abortion attitudes in US religious traditions, 1972-1998. Sociological Forum, 17(3), 397-422. doi:10.1023/A:1019627006778

Fiorina, M. P., Abrams, S. J., \& Pope, J. (2010). Culture war? New York, NY: Pearson Longman.

Flache, A., \& Macy, M. W. (2011). Small worlds and cultural polarization. The Journal of Mathematical Sociology, 35 (1-3), 146-176. doi:10.1080/0022250X.2010.532261

Gelman, A., \& Palmer, H. (2011). Polarization and issue consistency over time. Political Behavior, 33(2), $225-246$. doi:10.1007/s11109-010-9136-7

Hegselmann, R., \& Krause, U. (2002). Opinion dynamics and bounded confidence models, analysis, and simulation. Journal of Artificial Societies and Social Simulation, 5(3).

Hetherington, M. J., \& Weiler, J. D. (2009). Authoritarianism and polarization in American politics. Cambridge University Press.

Hirschl, T. A., Booth, J. G., Glenna, L. L., \& Green, B. Q. (2012). Politics, religion, and society: Is the United States experiencing a period of religious-political polarization? Review of European Studies, 4(4), 95-109. doi:10.5539/res. $\mathrm{v} 4 \mathrm{n} 4 \mathrm{p} 95$

Hunter, J. D. (1991). Culture wars. New York, NY: Basic Books.

Joan Estaban and Debraj Ray. (2012, April). Comparing polarization measures.

Kaner, H. C., Mohanty, S. G., \& Lyons, J. C. (1980). Critical values of the Kolmogorov-Smirnov one-sample tests. Psychological Bulletin, 88(2), 498-501. doi:10.1037/0033-2909.88.2.498

Mas, M., Flache, A., \& Helbing, D. (2010). Individualization as driving force of clustering phenomena in humans. PLOS Computational Biology, 6(10), e1000959. doi:10.1371/journal.pcbi.1000959

McCarty, N., Poole, K. T., \& Rosenthal, H. (2008). Polarized America: The dance of ideology and unequal riches. Cambridge, MA: MIT Press.

Page, B. I., \& Robert, Y. (1992). Shapiro. Chicago, IL: University of Chicago Press.

Smith, T. W., Marsden, P. V., Hout, M., \& Kim, J. (2013). General social surveys, 1972-2012. Retrieved from http:// sda.berkeley.edu/D3/GSS12/Doc/gs12.htm

Thomas, C. (2013). SDA: Survey documentation and analysis. Retrieved from http://sda.berkeley.edu/sdaweb/analysis/ ?dataset=gss 12

Wilcox, R. R. (1997). Some practical reasons for reconsidering the Kolmogorov-Smirnov test. British Journal of Mathematical and Statistical Psychology, 50(1), 9-20. doi:10.1111/bmsp.1997.50.issue-1

\section{Appendix-Data and analysis results}

The following tables present the data and polarization measurement values for the analyses presented above. The numbers for the counts of respondents in each each category were pulled from the SDA web resource accessing the GSS database. Count values are weighted using the SDA's composite weight variable. "This composite weight adjusts for number of adults in the household, for black oversampling in 1982 and 1987, and for problems with form randomization in 1978-1985. It does not adjust for differential nonresponse" (Smith et al., 2013). The measure values in these tables were calculated from the example measures presented in the body of the paper. Combined bin count data used for the endogenous group analysis is the sum of the three groups. All analyses in the paper use percentages calculated for each year from this SDA-corrected count data. 

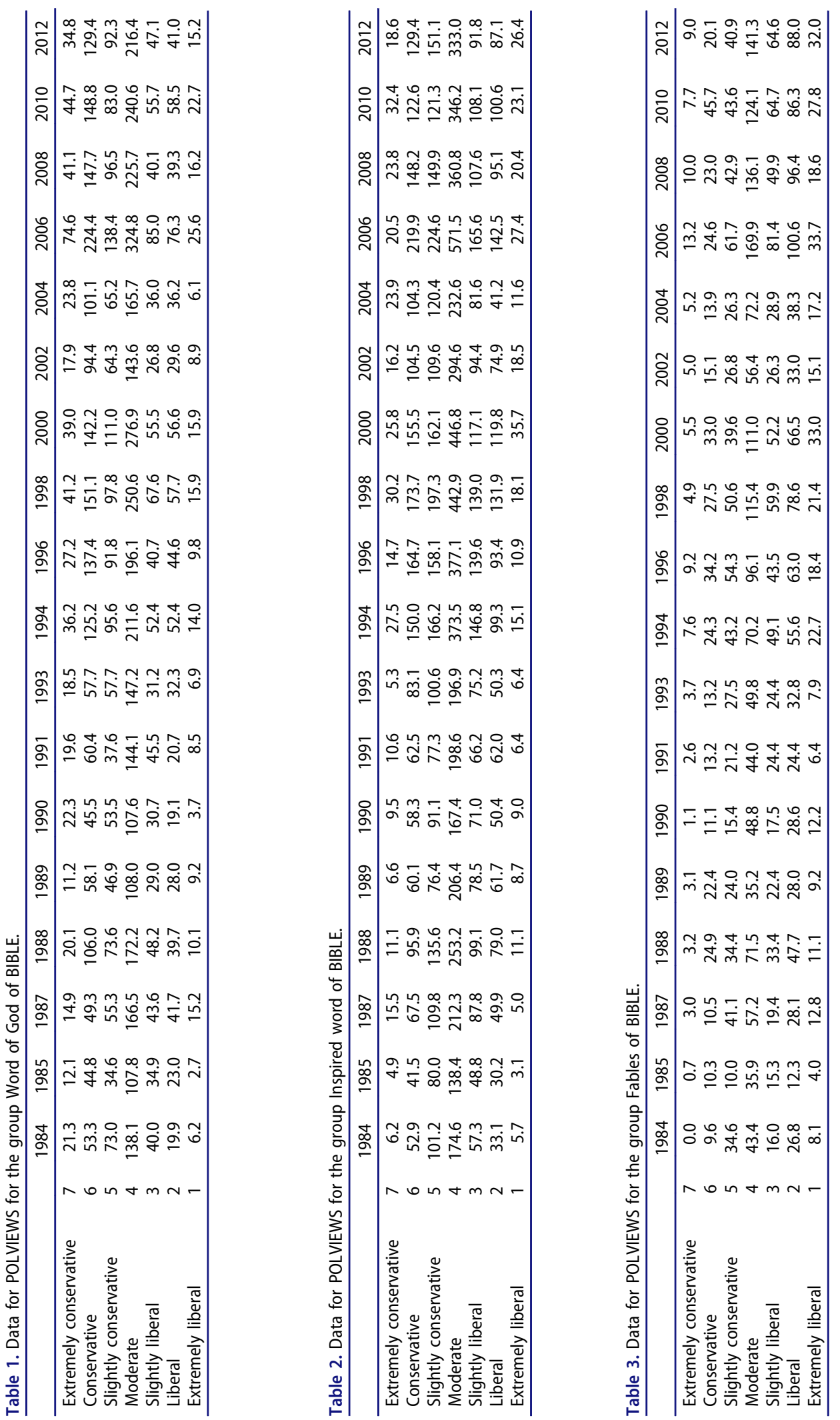

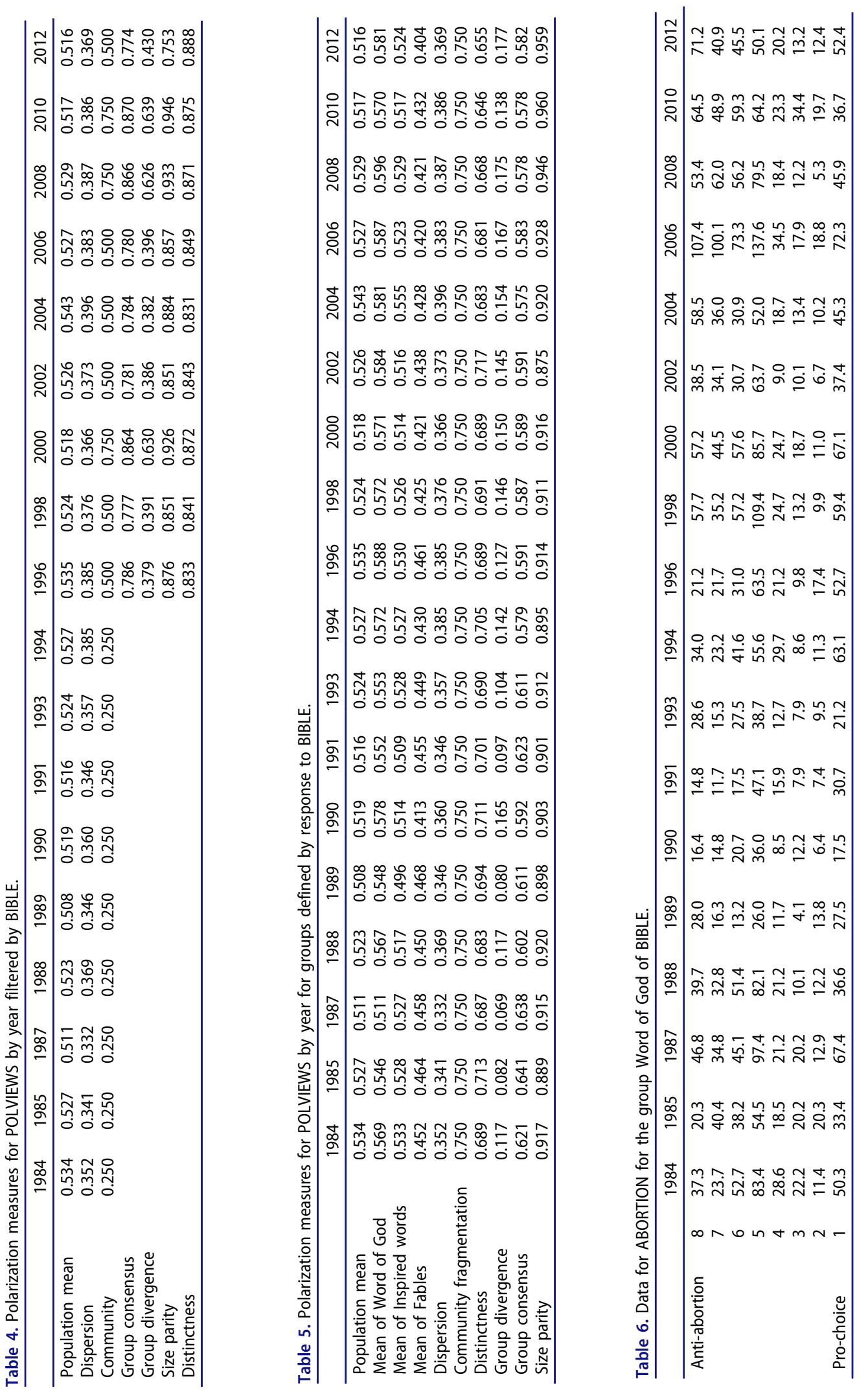

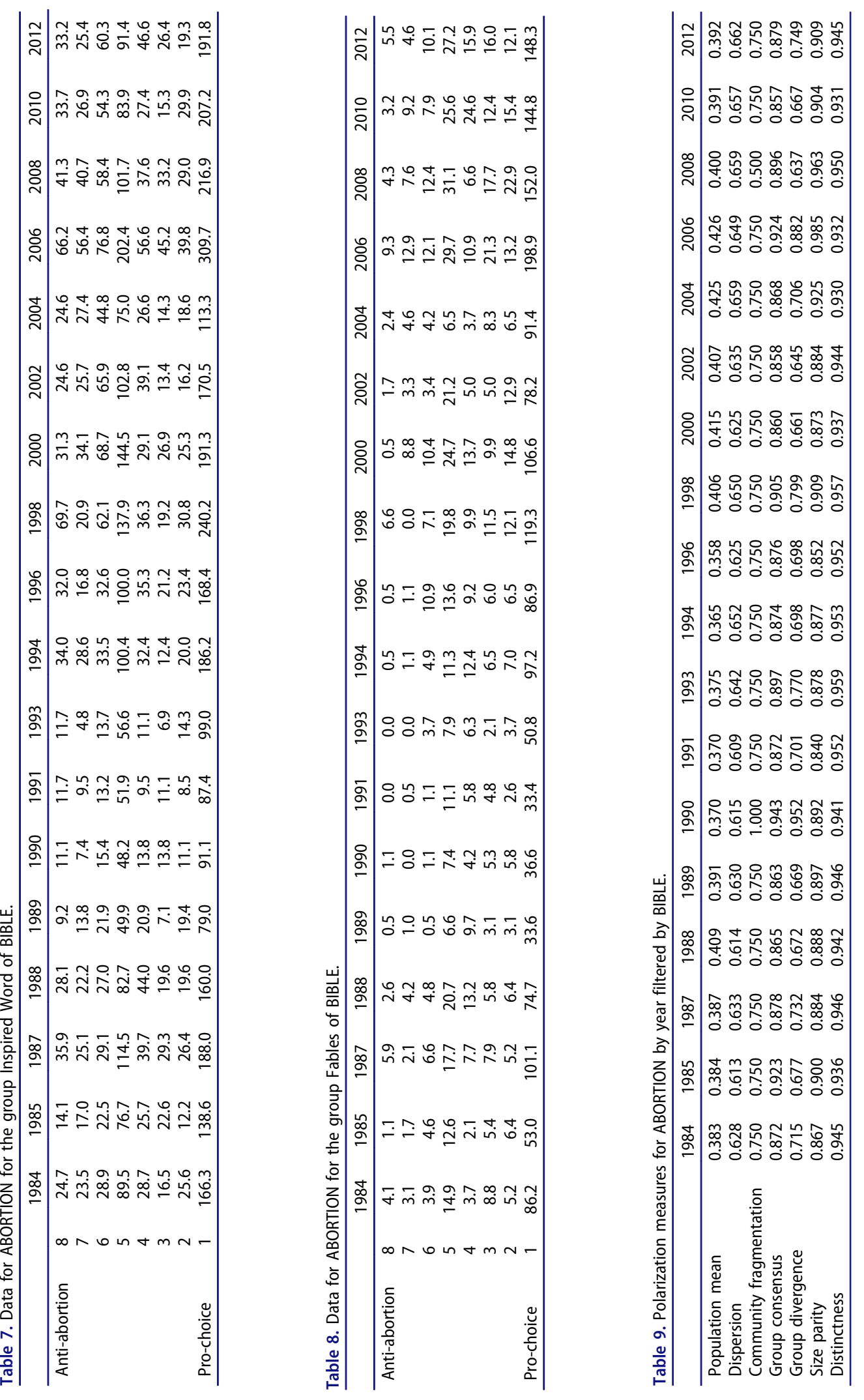

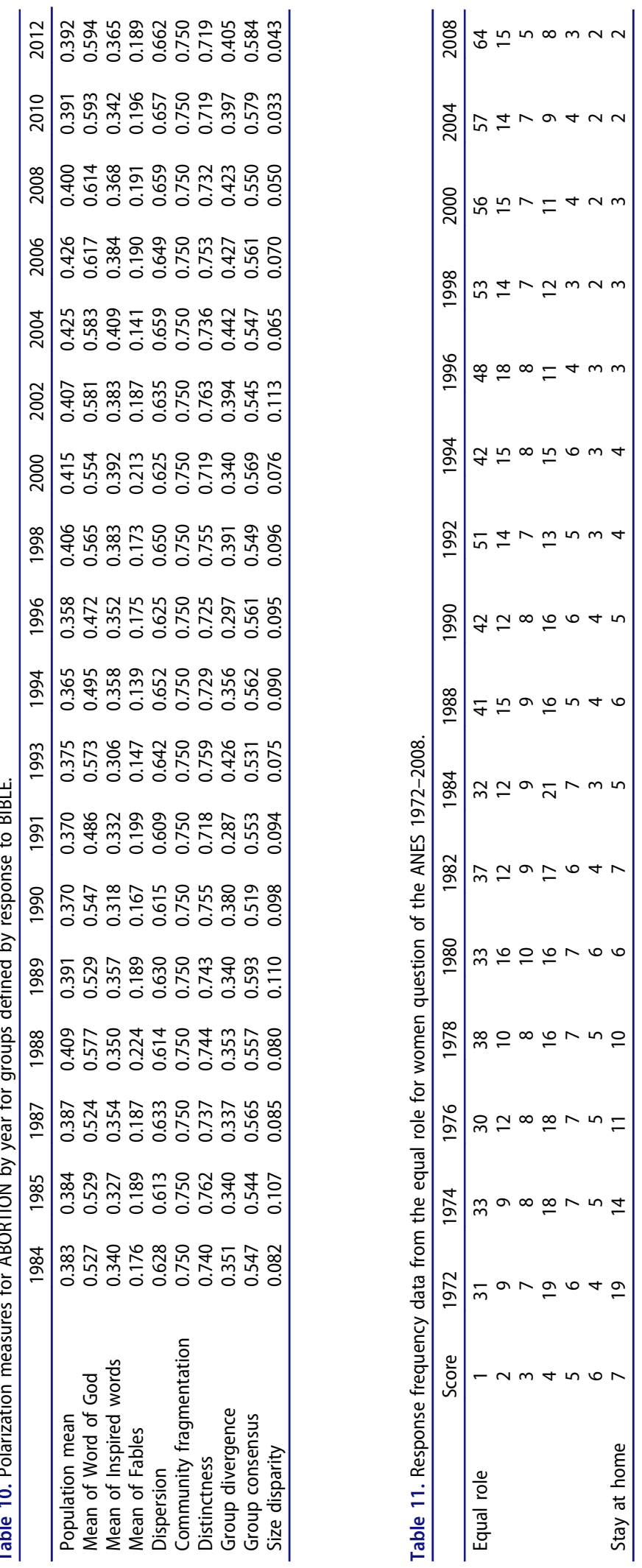